\title{
Zur Erinnerung an deutsche Opfer: Geschichte, Zeugnis und Fiktion in Grass' Novelle Im Krebsgang.
}

Helmut Galle*

\begin{abstract}
Analysing a novelette by G. Grass, the author points out a recent tendency of German literature on World War II. Several new books on this subject focalize, not on guilt, but rather on the suffering of German population. Grass's text shows a particular respect for historical facts and allows the reader to easily distinguish between factual and fictional elements. In the fictional parts the author imitates the genre of testimony and qualifies, by these means, the novelette for collective memory. The fictional action also contains political arguments that explain, why Germans should commemorate their victims: for the sake of truth and emotional balance, and in order to find a way out of the circuit of the violence, perpetuated through generations. Grass's book can be seen as part of a tradition of representing historical events, at the service of a humanistic and enlightened collective memory, which, in Germany, has its roots in Schiller's dramatic works.
\end{abstract}

Keywords: Fiction; History; Collective Memory.

Zusammenfassung: Der Artikel untersucht anhand einer Novelle von G. Grass eine aktuelle Tendenz der deutschen Literatur über den Zweiten

Der Autor ist Professor an der Universidade de São Paulo 
Q Weltkrieg, in der nicht mehr die Schuld, sondern das Leiden der deutschen

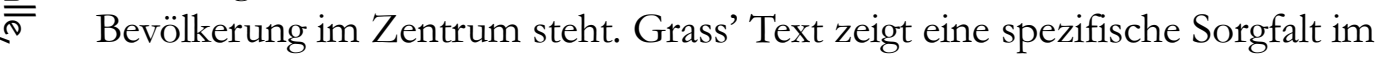
I Umgang mit den historischen Fakten und macht dem Leser die Differenz von 1 faktualen und fiktionalen Elementen besonders transparent. Im fiktionalen () Bereich imitiert der Autor die Gattung des Zeugenberichts, was wesentlich dazu beiträgt, seine Novelle zum Träger von kollektiver Erinnerung zu qualifizieren. In der fiktionalen Handlung ist außerdem eine politische Argu$\frac{0}{\bar{\sigma}}$ mentation aufgehoben, mit der unterstrichen wird, warum auch der deutschen इ Opfer gedacht werden muss: um der Wahrheit und des emotionalen 즈 Gleichgewichts willen und um einen - wenngleich schmalen - Ausweg aus 융 dem über Generationen fortgesetzten Teufelskreis der Gewalt zu finden. Grass' Novelle steht damit zugleich in einer Tradition von Vergegenwärtigung historischer Ereignisses im Dienste einer humanistisch-aufgeklärten kollektiven Erinnerung, das sich in Deutschland auf Schillers Dramen zurückverfolgen lässt.

Stichwörter: Fiktion; Geschichte; kollektives Gedächtnis.

Resumo: O artigo analisa, por meio de uma novela de G. Grass, uma tendência atual da literatura alemã sobre a II Guerra. Nesta nova literatura já não é mais a culpa, mas o sofrimento da população que se focaliza. $\mathrm{O}$ texto de Grass apresenta um cuidado particular nos fatos históricos, facilitando aos leitores distinguir entre elementos factuais e ficcionais. No âmbito da ação ficcional, o autor imita o gênero do testemunho, fato que contribui para qualificar sua novela como meio da memória colectiva. A ação ficcional desenvolve, além disso, uma argumentação política que explica a necessidade de comemorar também as vítimas alemães: para fazer jus à verdade, ao equilíbrio emocional e para encontrar uma saída do circuito da violência, perpetuada através das gerações. A novela de Grass encontra-se, por conseguinte, na tradição da representação de eventos históricos, a serviço de uma memória colectiva humanista e iluminada que, na Alemanha, remete à obra dramática de Schiller.

Palavras-chave: Ficção; história; memória colectiva. 


\section{Grass als Autor deutscher Schuld - und nun auch deutschen Leidens}

Für Friedrich Schiller bildete die europäische Geschichte noch ein großes Reservoir von Stoffen, aus dem er Vorwürfe wählen konnte, die geeignet schienen, seine Zeitgenossen zu humanisieren. In der deutschen Literatur seit 1945 ist die Beschäftigung mit den entfernteren Ereignissen ganz zurückgetreten hinter der dem einen großen Thema der Zeitgeschichte, das auch nach Vereinigung und Jahrtausendwende noch nicht in die geschichtliche Distanz gerückt ist, und dem eine ,Historisierbarkeit' sogar prinzipiell abgesprochen wird. Unter den literarisch anspruchsvollen deutschen Autoren, die sich mit dieser „Vergangenheit, die nicht vergehen will” (E. Nolte), diesem alles verschlingenden ,Schwarzen Loch' in der Mitte des 20. Jahrhunderts auseinandergesetzt haben, ist Grass sicherlich der international renommierteste. Seine „Danziger Trilogie” hat Eberhard Lämmert - neben den Werken von Solschenyzin und Szczypiorski - als eminenten deutschen Beitrag zur Phänomenologie des Totalitarismus hervorgehoben (LÄMMERT 1996). An diesen erzählerischen Komplex knüpft - 40 Jahre später - die Novelle Im Krebsgang (2002) an, freilich nimmt sie nicht mehr das soziale und zwischenmenschliche Geflecht der totalitären Gesellschaft ins Visier, in dem Menschen zu Tätern werden konnten. Im Vordergrund steht hier ein Ereignis gegen Ende des 2. Weltkriegs, das Deutsche nicht als Täter, sondern in der Rolle unschuldiger Opfer präsentiert. Dies ist, abgesehen von einzelnen Episoden in Mein Jahrbundert, neu bei Grass und ein Bruch mit den Konventionen der deutschen Linken, die das Thema ,deutsche Opfer' aus politischen Gründen jahrzehntelang totgeschwiegen hatte. In der Novelle wird es nun sogar als „bodenloses Versäumnis" angesprochen, dass der Autor und seine Generation das Elend der Flüchtlinge nicht dargestellt hätten, dass sie geschwiegen hätten „über soviel Leid, nur weil die eigene Schuld übermächtig und bekennende Reue in all den Jahren vordringlich gewesen sei" (Grass 2002: 99). In den

Vgl. auch die vom Autor in Reden und Interviews gemachten Aussagen Grass 2001, 2002b. 
Rezensionen wurde Grass allerdings weniger dieser vermeintliche Tabubruch vorgeworfen ${ }^{2}$ als vielmehr das ,Aufspringen' auf einen bereits von anderen Autoren vorweggenommenen Trend $^{3}$ sowie Schwächen der narrativen Konzeption. Es lasse epische Souveränität vermissen, dass der Autor sich über große Strecken nicht auf seine fiktionale Kraft verlasse, sondern Zuflucht sich anlehne bei den faktischen Ereignissen. ${ }^{4}$ Daher sei Im Krebsgang auch nicht geeignet, ,Vergangenheitsbewältigung' im Stile der „Danziger Trilogie" zu leisten, die nach wie vor als paradigmatisch für das Werk Grass' und die Literatur über die jüngere deutsche Geschichte angesehen wird.

Gegen solche Ansichten wird hier die These vertreten, dass die Veränderungen in der öffentlichen Kommunikation seit den sechziger Jahren Grass nicht nur das Thema, sondern auch die Art der Behandlung geradezu aufgenötigt haben. Das Bewusstsein von der Bedeutung des ,Zeugen’ für das kollektive Gedächtnis ${ }^{5}$ und eine neue Sensibilität für die Differenz von Fakten

2 Grundsätzliche Kritik an der Verschiebung / Ausweitung des Opferbegriffs auf die Täter war noch 2002 in den verspäteten Reaktionen von L. Norfolk, J. AdLer u.a. auf B. Schlinks Roman Der Vorleser in der englischen Presse laut geworden, was von W. Winkler in der Süddeutschen Zeitung aufgegriffen wurde, aber in deutschen Medien keine allgemeine Zustimmung fand.

3 Vgl. etwa das Spiegel-Interview mit Walter Kempowski, in dem dieser zu Recht auf sein monumentales Echolot-Projekt verweist; in den Bänden Barbarossa und Fuga Furiosa seines „kollektiven Tagebuch" haben die Stimmen deutscher Kriegsopfer einen substantiellen Anteil im gewaltigen Chor der Dokumente. Dass einzelne Autoren der Nachkriegsliteratur den Bombenkrieg gegen die Bevölkerung thematisierten, ist von HAGE 2003 dokumentiert.

4 Die Rezensenten von Im Krebsgang bemängelten meist die literarischen Qualitäten (BuCheli 2002, MAILER 2002, einschränkend FranZEN 2002), das Übergewicht der historischen Recherche gegenüber der ,artistischen, künstlerischen Gestaltung” (SPIEgel 2002), die aus der Novelle ein ,literarisch tapeziertes historisches Feature" mache (KNIPPHALs 2002) und die Tatsache, dass Grass in der literarischen Aufarbeitung von Flüchtlingselend und Bombenkrieg durchaus nicht der Tabubrecher sei, als den er sich darstelle (SPIEGEL 2002).

5 Vgl. RüsEN 2001: 232: „, Die Authentizität konstitutiver Erinnerungen, wie sie vor allem den überlebenden Opfern zugebilligt wird, ist eine metahistorische Größe. Diese Methahistorizität wird mit dem Begriff des Mythischen zum Ausdruck gebracht. Da aber im normalen Sprachgebrauch mythisch als nicht-historisch verstanden wird, ist diese Bezeichnung irreführend. Diese Authentizität 
und Fiktionen ${ }^{6}-$ trotz und gerade aufgrund der poststrukturalistischen Grenzverwischungen - haben den Autor zu einer Erzählstrategie bestimmt, die sich funktional zu seiner Intention verhält: die Leiden deutscher Opfer in das Gedenken einzubeziehen.

Zur Stützung dieser These soll - nach einer Übersicht zu Inhalt und Struktur der Novelle (2) - untersucht werden, mit welchen narrativen Strategien der Autor die Faktenebene innerhalb der Fiktion als solche intakt lässt (3), wie der Zeugnischarakter hergestellt wird (4), welche Funktion die neonazistischen Internetpropaganda im Kontrast hierzu erhält (5) und wie sich Grass' Text innerhalb des deutschen Gedächtnisdiskurses nach 1990 ausnimmt (6).

\section{Inhalt und narrative Struktur}

Die Handlung der Novelle umkreist einerseits die historischen Ereignisse des Untergangs der „Gustloff” und, ebenso historisch dokumentiert, die Biographien der Akteure Wilhelm Gustloff (Nazi-Statthalter in der Schweiz), David Frankfurter (Mörder Gustloffs mit politischer Motivation) und Alexander Marinesko (russischer U-Boot-Kommandant, verantwortlich für die Versenkung des Schiffes).

Diese prinzipiell ,bekannten' Vorgänge werden hier in der Fiktion als Ergebnisse der Recherche des Erzählers Paul Pokriefke dargestellt. Dieser

ist prähistorisch, aber nicht nicht-historisch, im Gegenteil: Sie geht als notwendiges Element der historischen Sinnbildung in die Historizität selbst ein.” Zur „Deutungsautorität” des Zeugentextes für die kollektive Erinnerung auch ReEMtsma 2002: 230.

$6 \quad$ Vgl. dazu Marcel Beyer, der für die neuartige, seit den neunziger Jahren entstehende Literatur über die Nazizeit steht, und Kritik an den ungenauen Opferzahlen in K. Vonneguts Dresden-Roman übt. „,...] Slaughterbouse-Five hat in meinen Augen als Roman jeden Wert verloren. Als wäre es nicht ein Werk der Fiktion, sondern ein Werk der Fälschung - und das, obwohl der Autor doch aufrichtig versucht hat, sich vom Fiktiven zum Faktischen hin zu bewegen." BEYER 2003: 300. - Zur bereits in der Blechtrommel erkennbaren Rücksicht auf historische Faktizität (vor allem im Kapitel über die Verteidigung der polnischen Post) s. Mazzari 1999: 118 ff. 
ist Sohn von Tulla Pokriefke, einer Protagonistin aus Grass' Danziger Trilogie, deren Biographie hier fortgesetzt wird. Sie war hochschwanger auf der „Gustloff” aus Danzig geflüchtet, wurde bei deren Untergang gerettet und unmittelbar darauf von einem Kind, dem Erzähler, entbunden, dessen Geburt also mit der Katastrophe zeitlich zusammenfällt. Er gibt nun in Rückblicken Hinweise auf seine generationstypische Nachkriegskarriere als Journalist in Westberlin und das weitere Ergehen seiner Mutter, die in Schwerin / DDR bleibt. Parallel zu seinen historischen Ermittlungen über den Untergang der „Gustloff” wird diesem Paul Pokriefke allmählich klar, dass die rechtsradikale Internetseite, auf die er bei seinen Recherchen immer wieder stößt, ausgerechnet von seinem Sohn Konny unterhalten wird. Dieser Enkel ist auf seine Weise der Forderung der Großmutter Tulla nachgekommen, vom Untergang der Gustloff müsse Zeugnis abgelegt werden, nachdem sein Vater Paul sich genau diese Wunsch der Überlebenden so lange hartnäckig verweigert hatte. Im Internet entspinnt sich eine Kontroverse zwischen dem Neonazi Konny und dem gleichaltrigen David, der sich als Jude mit antifaschistischen Überzeugungen darstellt. Als die beiden dann real aufeinandertreffen, wird David von Konny erschossen; die im Internet behauptete jüdische Identität stellt sich nun als antifaschistische Mimikry heraus: „David” war tatsächlich ein Wolfgang aus deutsch-christlichem Hause. Konny erhält eine Gefängnisstrafe. Weil der Vater jetzt endlich seinen Bericht - den der Leser mit der vorliegenden Novelle identifizieren darf - verfasst hat, in dem die Katastrophe der "Gustloff”, die Geschichte der Mutter Tulla und die Tat des Sohnes enthalten sind, ist nun der Weg für eine Annäherung der drei Generationen geöffnet. Doch im Internet agiert bereits ein ,nationaler' Freundeskreis für den neuen „Märtyrer” Konny Pokriefke und kündigt damit an, dass der Teufelskreis der Gewalt nicht zu Ende geht.

Im Text lassen sich folgende, miteinander verwobenen, narrativen Ebenen ausmachen, von denen eine die Rahmenhandlung abgibt, drei die Schiffskatastrophe unterschiedlich perspektivieren, und eine fünfte dem Autor metanarrative Botschaften ermöglicht:

1. Des Erzählers autobiographische Einlassungen, seine Recherchetätigkeit und der Schreibprozess; durch die Stimme des Erzählers werden alle Handlungen und Dokumente an den Leser vermittelt und kommentiert; er sieht sich selbst jedoch bloß als Berichterstatter. 
2. Fiktionales Zeugnis Tulla Pokriefkes über ihre Fahrt auf der „Gustloff” von Danzig nach Schwerin. Die Aussagen werden in wörtlicher Rede in die Novelle eingestreut, z.T. auch vom Erzähler indirekt referiert; sie betreffen insbesondere die letzten Augenblicke der „Gustloff” und die Rettung der Überlebenden.

3. Bericht über David Frankfurter, Wilhelm Gustloff und Alexander Marinesko; der Erzähler referiert Faktenmaterial zum Schiff aus den tatsächlich publizierten Printquellen und Filmen zum Thema.

4. Fiktive neonazistische Propaganda über die „Gustloff” im Internet.

5. Metanarrative Einschübe, in denen der Erzähler Kommentare des „Alten" referiert, die sich dem Autor Grass zuordnen lassen. ${ }^{7}$

Schematisch ließen sich diese Ebenen folgendermaßen darstellen: ${ }^{8}$

\begin{tabular}{|c|c|c|c|c|c|}
\hline & $\begin{array}{c}1 . \\
\text { Rahmen- } \\
\text { erzählung }\end{array}$ & $\begin{array}{c}2 . \\
\text { Zeugnis }\end{array}$ & $\begin{array}{c}3 . \\
\text { Bericht }\end{array}$ & $\begin{array}{c}4 . \\
\text { Propaganda }\end{array}$ & $\begin{array}{c}. \\
\text { metanarrativer } \\
\text { Kommentar }\end{array}$ \\
\hline Modus & fiktional & fiktional & faktual & fiktional & faktual \\
\hline Fokalisierung & intern & intern & extern & „Null“ & extern \\
\hline Beziehung & autodiegetisch & autodiegetisch & heterodiegetisch & heterodiegetisch & heterodiegetisch \\
\hline Ebene & extradiegetisch & intradiegetisch & intradiegetisch & intradiegetisch & extradiegetisch \\
\hline Generation & Zweite & Erste & Zweite & Dritte & Erste \\
\hline
\end{tabular}

Dem „Alten” liegt die Figur Tulla Pokriefke so väterlich am Herzen, wie es ihrem literarischen Schöpfer wohl ansteht. Er gibt die Anweisungen für die Gestaltung der Novelle und bezeichnet sich als prädestiniert für dieses Thema. Insofern lässt sich zwar nicht behaupten, hier spräche unverstellt der Autor, denn er wird ja nur durch eine fiktionale Quelle vermittelt. Aber da die Figur des "Alten" so gestaltet ist, dass der Leser den Autor darin erkennen muss, dürfen wir schließen, Grass wolle, dass der Leser ihm die hier referierten Ansichten zuschreibt. Im übrigen hat Grass in Interviews die Autorfigur im Text mit der ersten Person Singular belegt: „Reizvoll war für mich der im Buch mitschwingende Streit zwischen dem fiktiven Erzähler und dem Autor, meiner Person. Ich halte das Ganze für eine Novelle, er besteht darauf, einen Bericht zu schreiben.” GRAss 2002c. Der „Alte” hält sich freilich an seine fiktionalen Rolle, 
$\stackrel{\Omega}{\underline{\rho}}$ Der Text präsentiert die historischen Ereignisse um den Untergang der

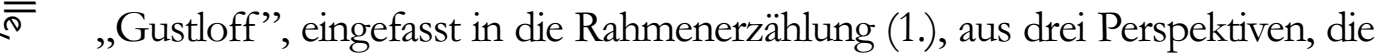
I jeweils anderen Diskurs- und Rezeptionsmodi gehorchen. Der faktuale Bericht (3.) von den objektiven Umständen erscheint in der historiographischen Modalität mit konsequent externer Fokalisierung aus der Perspektive der zweiten Z Generation (Paul Pokriefke). Die Stimme des fiktiven Erzählers ist das Medium, das die vom realen Autor recherchierten Fakten an den Leser vermittelt. $\frac{\sigma_{\bar{N}}}{\bar{\sigma}}$ Das Zeugnis (2.) gibt die subjektive Erfahrung der Überlebenden Tulla (erste $\bar{\xi}$ Generation) als fiktive Autobiographie aus der Innenperspektive wieder. Ihre 중 Aussagen werden zwar auch durch den Erzähler mitgeteilt und stehen nicht in einer separaten Sequenz, sie werden aber immer wieder durch wörtliche Rede herausgehoben. Die Internetproganda (3.) ist weitgehend aus der kommentierenden Perspektive des Erzählers wiedergegeben, steht im fiktionalen Modus wie (2.) und stellt ebenfalls die historischen Ereignisse (intradiegetisch) dar; der fiktive Erzähler Konny (dritte Generation) beansprucht, dass seine Internetseite als die eigentliche historische Wahrheit (faktualer Modus) zu ansehen sei. Die Sequenzen, in denen der „Alte” zu Wort kommt (5.), ermöglichen die Einschaltung metanarrativer Kommentare des Autors in die fiktionale Erzählung und tragen wiederum faktualen Charakter, was ihren Status als Aussagen des Autors über seinen Stoff angeht; das Verhältnis des Erzählers zum Autor ist ,natürlich' fiktional strukturiert. Die Ebenen 3 und 5 referieren auf ein und dieselbe Welt, die mit der physischen Welt der Leser identisch ist, die Ebenen 1, 2 und 4 referieren auf eine fiktionale Welt, in der (wie im historischen Roman) die historischen Fakten als Skelett fungieren: Innerhalb des historisch Gegebenen bewegt sich die fiktionale Handlung als mögliche und symbolisch allgemeingültige.

\section{Die faktuale Ebene des Berichts}

Die Differenz zwischen fiktionalen und faktualen Aussagen ergibt sich daraus, dass die Familie Pokriefke und alles, was ihr im Laufe der Handlung

wenn von er vom Erzähler Details über Tulla verlangt oder wenn er behauptet, er habe „nicht wissen können”, dass Tullas Enkel hinter der neonazistischen Internetseite stehe. (78)

$8 \quad$ Zur Terminologie GenetTe 1998 und 1992. 
zustößt, Produkte der Phantasie von Günter Grass sind, während David Frankfurter den Mord an Wilhelm Gustloff (ausweislich seines eigenen Geständnisses und des Schweizer Strafprozesses) wirklich begangen hat, auch der russische U-Bootkommandant und sein Angriff auf das deutsche Schiff sind historische Tatsachen, die durch das Wrack, Augenzeugen- und Marineberichte verbürgt werden. Nun ließe sich argumentieren, dass eine Aussage aus dem Munde eines fiktionalen Erzählers per se keine Aussage mit referentiellem Status sein kann. Man könnte daher meinen, im Rahmen der Novelle sei eine Aussage wie

zum Landesgruppenleiter jedoch war er [W. Gustloff] von Gregor Strasser ernannt worden

eine fiktionale Aussage, weil sie aus der Feder des imaginären Paul Pokriefke stammt und nicht vom Autor selbst verantwortet werde. ${ }^{9}$ Andererseits hat der Autor die Faktizität dieser Ebenen so transparent gestaltet, dass sich die Darstellung anhand des dokumentarischen Materials der Archive verifizieren / falsifizieren lässt wie die Aussagen eines Historikers. Der Leser kann erkennen, wo er es mit solchen referentialisierbaren Aussagen über historische Personen zu tun hat und wo die ebenso deutlich als Fiktion markierte Handlung der Grass' schen Figuren wieder einsetzt. Hier zeigt sich eine deutliche Differenz zu der mimetischen Funktion von Aussagen wie etwa die von Käte Hamburger zitierte Passage aus Tolstois Krieg und Frieden

Um halb sechs Uhr ritt Napoleon nach dem Dorfe Schewardino. Es wurde schon hell; der Himmel hatte sich aufgeklärt; eine einzige Wolke hing noch im Osten.

Hier sind die Aussagen eingepasst in die fiktive Handlung und stellen eine mimetische Szene her, in der Napoleon, seine Handlungen und deren

9 Alfred Döblin beispielsweise hat den historischen Roman dagegen verwahrt, im Modus der Historiographie gelesen zu werden. „Der historische Roman ist in erster Linie ein Roman und in zweiter - keine Geschichte.” DöBLIN 1963: 169. 
Motive für den Leser erkennbar der auktorialen Erzählerstimme entstammen (Vgl. Hamburger 1957: 61). In Grass' Novelle hingegen bleibt die fiktive Erzählerinstanz dank der wahrnehmbaren Quellen nur ein Durchgangsmedium zu der von Leser und Autor geteilten Sphäre historischer Wirklichkeit.

Es spielt dabei keine Rolle, ob der Leser über das historische Geschehen bereits in dem Maße informiert ist, dass er die Wahrheit jeder einzelnen Bemerkung des Erzählers beurteilen kann. Es genügt, dass im Text die Signale eindeutig gestellt sind und dass der Autor so mit seinem Namen dafür einstehen muss, wenn er etwa nachprüfbar die Rolle Frankfurters abweichend von den gängigen Untersuchungen dargestellt oder auch nur Elemente hinzuerfunden hätte, die nicht von den verfügbaren Dokumenten gedeckt wären. ${ }^{10}$ Es wurde bereits darauf hingewiesen, dass der Autor sich selbst als Figur in die Fiktion hineingeschmuggelt hat und auch auf diese Weise die Fiktionalität des Erzählers relativiert.

Für die Ebene der historischen Faktizität gilt damit ein anderer Rezeptionsmodus als für die Fiktion. Es besteht - nach dem gegenwärtigen Stand der Erkenntnis - kein Zweifel daran, dass der erste Torpedo traf, dass die „Gustloff” untergegangen ist, dass die meisten Kinder umkamen. Es gibt auch keinen Anlass, die Existenz des Autors Grass zu bezweifeln und zu unterstellen, dass er die ihm von seinem Erzähler in der Novelle zugeschriebenen Meinungen nicht auch in unserer realen Welt vertreten würde. ${ }^{11}$ Während wir die fiktionalen Figuren und ihre Handlungen kritisch daraufhin prüfen, ob sie 'in sich stimmig', wahrscheinlich und repräsentativ sind (die Kritik gilt dann letztlich ihrem Schöpfer), ${ }^{12}$ verläuft die Wahrnehmung

10 Dorrit Cohn plädiert (mit W. Turner) im Falle eines (gattungstypischen) Romans wie Tolstois Krieg und Frieden dafür, dass die exakten historischen Daten und abgesicherten Aussagen, die er enthält, vom Leser auch als solche erkannt werden; selbst nach der Lektüre von postmodernen Parodien, ,we do tend to approach this genre differently from other novelistic genres." COHN 2002: 159

11 Vgl. Grass 2001, 2002b, 2002c.

12 Der Mord Frankfurters an Gustloff ist nicht in derselben Weise kritisierbar, wie der Mord Konnys an David. Die im ersten Fall nur referierten Motive können wir dem Täter als schlechte Ausrede anrechnen, im zweiten Fall würden wir sie dem Autor als schlecht erfunden vorwerfen. Die erste Version findet in unserem 
der faktualen Ebene in Etappen: am Anfang steht die Frage, inwiefern sich der Autor überhaupt an die Geschichte gehalten hat. Ist dies einmal entschieden, würde die Rezeption der mitgeteilten Elemente jedoch in zwei verschiedenen Bahnen verlaufen: Das Verhalten der fiktionalen Figuren Paul, Konny und Tulla Pokriefke steht als solches zur Diskussion und kann dem Autor als gut oder weniger gut imaginiert angeschrieben werden. Der Leser sieht darin mehr oder weniger repräsentative Vertreter der jeweiligen Generation. Dagegen lassen sich die Figuren Frankfurter, Gustloff und Marinesko und ihre Handlungen bzw. das Urteil der Schweizer Justiz nicht wie Phantasien des Autors kritisieren, sondern allenfalls die Art und Weise, wie sie hier zusammengeführt und mit der Fiktion konfrontiert sind. Der Leser kann aber aus den mitgeteilten Ereignissen um den Untergang der „Gustloff” auch andere Schlüsse ziehen als die Figuren der Erzählung oder der Autor, dazu stehen ihm hier genug Daten zur Verfügung. Die fiktionalen Passagen lassen kein solches unabhängiges Urteil über die Geschichte zu; wenn der Leser sie nicht plausibel findet, kann er sie allenfalls verwerfen.

Es soll im Rahmen dieser Analyse nicht diskutiert werden, inwiefern die im Gefolge von Poststrukturalismus und New Historicism aufgekommene Einebnung der epistemologischen Differenz von historiographischem und literarischem Diskurs haltbar ist. ${ }^{13}$ Für Grass und die große Mehrheit seiner

weiteren Weltverständnis Beachtung als denkbare Verhaltensweise in bestimmten Situationen. Die zweite wird gelöscht bzw. gebucht als nicht anschlussfähige fremde Phantasie.

13 Stellvertretend für viele andere skeptische Stimmen sei hier Hans Ulrich Gumbrecht zitiert: „Die gravierenden Probleme beginnen, sobald das Pochen auf die Subjektivität der Historiker zur Aufkündigung der Prämisse führt, es gebe eine Realität jenseits dieser Subjektivität - und damit zur Abschaffung des Wunsches, diese Realität zu erreichen (eines Wunsches, der ebenso unerfüllbar ist, wie alle anderen Wünsche). Sobald sich der Neue Historismus dieses Wunsches entschlägt, unterscheidet er sich nicht mehr von der Literatur und kann daher nie an die Stelle des traditionellen - durch Anspruch auf realweltlichen Bezug begründetet - historiographischen Diskurses treten." GUMBRECHT 2001: 453. Zur fachlichen Kritik an Hayden White vgl. z.B.: OexLe 1992, 2000. Die grassierende Destruktion von Gattungsgrenzen in der Literaturtheorie wird von den tatsächlichen Mechanismen der Literaturrezeption regelmäßig dementiert. 
Leser darf davon ausgegangen werden, dass sie eine historische Realität für gegeben halten, welcher sich der Historiker mit den kritischen Methoden seines Faches und einem Fundus an reflektierten narrativen Strategien zu nähern versucht. Ohne diese Vorannahme wäre Grass’ Buch sinnlos.

Der Autor ist sich jedoch der Tatsache bewusst, dass er dem Leser eine Kontrolle darüber einräumen muss, welche Quellen er in welcher Weise für seine literarische Konstruktion verwendet hat. Er tut dies zwangsläufig, mit Hilfe seiner Figur Paul Pokriefke, der aber vom „Alten” streng angehalten wird, in diesem Bereich nicht auf die Kompetenzen eines auktorialen Erzählers zurückzugreifen: Er gestattet ihm weder die interne Fokalisierung der historischen Personen ${ }^{14}$ noch die imaginative Ausschmückung der historischen Daten; wo dies dennoch geschieht, ist es als gesteuerte Phantasie markiert. $^{15}$

Weder Gedanken noch Gefühle von Frankfurter, Gustloff und Marinesko werden mitgeteilt, wohl aber deren dokumentierte Selbstaussagen über Motive und Ziele. Wenn der Erzähler ausgewählte Informationen über den Hintergrund der zeitgeschichtlichen Ereignisse darstellt, vor denen sich Frankfurters Mord an Gustloff anbahnt, tut er dies im Rückgriff auf die damaligen Meldungen der (archivierten) Tageszeitungen, die Frankfurter tatsächlich gelesen haben kann. Erst auf einer letzten Ebene von szenischen Details finden sich fiktionale Elemente, die aber wiederum vom Erzähler

Vgl. hierzu Verf. über die Fälle Wilkomirski, Koeppen und Kolitz, GALLE 2001a, 2001b, 2002a und DieKmann 2002. Generell hat sich gezeigt, dass sich die poststrukturalistische Kritik an der Referentialität von Historiographie zusehends relativiert, sobald die zentrale Katastrophe des 20. Jahrhunderts in Frage steht, hierzu insbesondere die Aufsätze in FrIEDLANDER 1992.

14 Am Anfang des 9. Kapitels gibt der Autor seinem Erzähler Anweisung, seinen Figuren nicht in den Kopf zu schauen (nicht einmal seinem eigenen, fiktionalen Sohn) und sich an die verifizierbaren Fakten zu halten. Grass 2002: 199.

15 Vgl. die Passage, in der Paul Pokriefke ,nur spaßeshalber und um mich auszuprobieren" sich die Lizenz dazu gibt, eine Nazi-Pressekonferenz zur Einweihung des Schiffes als Prosopopoeie auszumalen. Grass 2002: 58 ff. Auf ähnliche Weise wird bei der Schilderung des Untergangs angedeutet, wie hier eine ausschmückende „Story” eingefügt werden könnte, um ein solches Procedere dann sogleich zu verwerfen. (GRAss 2002: 139) 
als solche bewusst gemacht werden, so etwa die Schilderung der Schüsse auf Wilhelm Gustloff, bei denen Frankfurter in der Erzählerversion den Hut auf den Knien hat, während er ihn in der später referierten Filmversion auf dem Kopf trägt, und diese beiläufige Differenz explizit thematisiert wird (GRAss 2002: 27f., 68).

Mit derselben Sorgfalt weist der Erzähler den Leser darauf hin, dass er der auktorialen Versuchung nicht nachgegeben hat, den Untergang des Schiffes durch erfundene Anekdoten episch auszumalen, wie in den gestellten Szenen des Films - und gibt ein ironisches Beispiel dafür, wie solche literarische Eigenmächtigkeiten aussehen würden. ${ }^{16}$ Noch was die Wahrscheinlichkeit einer Geburt des Erzählers unmittelbar nach der Rettung Tullas angeht, fügt er die im zeitlichen Umfeld bezeugten Fälle von Entbindungen an. An diesen Signalen entlang findet der Leser seinen Weg zwischen Fiktion und Historischem.

Die diegetische Ebene der Ereignisse um die Schiffskatastrophe, an die sich der Erzähler ,im Krebsgang' herantastet, ist keine Welt multipler Möglichkeiten. Sie ist festgelegt durch die Eckpunkte des historischen Geschehens und die dokumentierten Fakten der Biographien von David Frankfurter, Wilhelm Gustloff und Alexander Marinesko. Dies wird durch die kontinuierliche Kontrastierung mit den Eigenmächtigkeiten der neonazistischen Internetseite noch unterstrichen. Weit mehr als das Zeitkolorit, das der historische Roman von seinem jeweiligen Hintergrund erhält, weit mehr auch als die historischen Referenzen in der „Danziger Trilogie", sind die geschichtlichen Sachverhalte zentraler Inhalt der Novelle. Die Biographien der drei realen „Protagonisten” werden über große Zeiträume verfolgt, bis zu den Zeitpunkten, an denen sie sich kreuzen und darüber hinaus.

Das ist bemerkenswert, denn in einem streng historiographischen Sinn haben die persönlichen Schicksale der drei so viel mit der Versenkung eines mit Flüchtlingen beladenen deutschen Schiffes durch ein russisches U-Boot zu tun, wie nahezu alle anderen damals in diesem Feld agierenden Menschen. Insbesondere Frankfurter und Gustloff sind mit der Katastrophe lediglich

16 s. die vorangehende Fußnote. 
durch die zufällige Namensgebung verknüpft - das Schiff hätte anders getauft werden können. Auch bleibt ungewiss, ob nicht auch irgendein anderer russischer U-Boot-Kommandant anstelle Marineskos, das zivile Ziel „Gustloff” torpediert hätte .

Dass diese Lebensläufe erzählt werden, weist auf die Absicht des Autors, hier sein Bild von der Geschichte einfließen zu lassen; es handelt sich also um eine Sinngebung der Fakten, die wieder in eine fiktionale Richtung geht, weil sie nicht auf der Textoberfläche ausgewiesen und durch rationale Argumentation begründet ist. Dennoch handelt es sich nicht um eine Manipulation des Materials, sondern um ein legitimes - literarisches! Verfahren, geschichtlichen Erfahrungen zur Evidenz zu verhelfen.

Gerade die Fernperspektive auf die Lebensläufe von Frankfurter, Gustloff und Marinesko hebt nämlich jene Zufälligkeit hervor, mit der Menschen zu Tätern bzw. Opfern werden können. Die Zufälligkeit, als Jude geboren zu sein, bringt Frankfurter erst dazu, einen politischen Mord an einem Nazi-Funktionär zu erwägen und durchzuführen. Gustloff ist ein eher unauffälliger Nazi, der zudem, über seinen Mentor Gregor Strasser, mit dem von Hitler eliminierten „linken Flügel” der Partei verbunden scheint. Da er ,zufällig' in der Schweiz stationiert ist, bietet er sich dem Attentäter als leichtes Ziel an. Aber genaugenommen ist auch diese Zufälligkeit eine Folge seines Lungenleidens: Der Nazi wurde Bewohner des ,Zauberbergs", den Hans Castorp hatte verlassen müssen. Marinesko wird nicht durch besondere kriegerische Eigenschaften in die Täterrolle manövriert, sondern durch seine laxe Dienstauffassung, ${ }^{17}$ die ihn schließlich zu einer Kompensationshandlung zwingt und dann doch nicht mit der erhofften staatlichen Anerkennung belohnt wird; ob sein Verschwinden im Gulag überhaupt mit dem Abschuss der Gustloff zu tun hat, oder den inneren (Un-)Logik der sowjetischen Selbstvernichtung folgt, bleibt offen.

Diese Darstellungsweise suggeriert nicht, dass diese Menschen determiniert gewesen wären: Ohne eigene Entscheidungen hätte Frankfurter den Mord nicht begangen, Gustloff hätte nicht in der NSDAP Karriere

17 Das ist teilweise als Klischeevorstellung vom 'Wodka liebenden Russen' beanstandet worden, dürfte aber eher im Gegenteil als Relativierung von Täterklischees beabsichtigt sein. Ein historisches Detail kann eben gerade nicht 'Klischee' sein. 
gemacht, Marinesko nicht auf dieses konkrete Ziel feuern lassen. Aber die Entscheidungen werden in einen Kontext gestellt, der die Kontingenzen stärker in den Blick rückt und ein vorschnelles Urteil a posteriori problematischer macht. Es sei dabei auch nicht übersehen, dass sich hier die klassische Konstellation verkehrt: der Jude ist der Täter, ${ }^{18}$ der Nazi das Opfer und der Russe beides zugleich. Der Erzähler enthält sich denn auch der moralischen Kommentare und gibt eine relativ unparteiische Beschreibung der Handlungsketten. ${ }^{19}$ Sechzig Jahre nach dem Krieg kann der Leser damit die Freund-Feind-Zuweisungen (in diesem konkreten Fall) hinter sich lassen und sich der Katastrophe jenseits von Schuldzuweisungen zuwenden, die - im Falle der deutschen Leser - letztlich doch immer an die eigene Adresse zurückgehen müssten.

Vor dem Panorama dieser drei Biographien erscheint auch das - immer schon mit dem Bericht parallel geführte - Schicksal der fiktiven Tulla Pokriefke einer moralischen Beurteilung entrückt. Zwar wurde sie in Hundejabre und Katz und Maus bereits mit einer gewissen Ambivalenz ausgestattet, blieb aber doch eindeutig identifizierbar als Repräsentantin des Täterkollektivs, ein weibliches Pendant zu Walter Matern, und wie dieser wurde sie in ihrem marginalen Lebensbereich schuldig an einem Mitmenschen, an dem Gewalt zu üben ihr die neuen Machtverhältnisse plötzlich gestatten. Auch im Krebsgang bleibt sie eine schillernde Persönlichkeit, bewährt sich aber unter den gesellschaftlichen Bedingungen der DDR als unauffälliger und wertvoller Mitbürger. Diese Bewährung und das Überleben der Schiffskatastrophe rehabilitieren sie nun in der Fiktion - sogar in den Augen ihres ehemaligen Opfers. Was die jugendliche Tulla, jener „kalt und böse funkelnde Kobold” (Hubert Spiegel), anrichtete, war

18 Die Worte Frankfurters im Film von Lyssi, lassen ihn sogar die Ausdrucksweise der Nazis übernehmen, wenn er vom „Bazillus”, den er „treffen” wollte. (GRAss 2002: 69)

19 Die Vorgänge im U-Boot, die schließlich direkt zum Verderben von nahezu 10.000 ,unschuldigen' Menschen führen, werden mit einer gewissen ironischen Einfärbung, aber ohne alle Hassgefühle geschildert. Der Erzähler bangt mit den Russen um die Entschärfung des dritten, stecken gebliebenen Torpedos und bemerkt schließlich: „Zum Glück [für die U-Boot-Besatzung] fielen keine Wasserbomben. (GRASS 2002: 133) 

@ zwar „böse”, aber eigentlich doch nur „Mutwillen”, meint sogar das misshandelte Zigeunerkind Jenny Brunies, die nun wieder als Freundin Tullas im fernen West-Berlin als „Tante Jenny” Anteil am Werdegang von Paul Pokriefke übernimmt. ${ }^{20}$
Der Autor hat durch diese Komposition seiner Auswahl von historischen Personen und fiktionalen Symbolfiguren eine Diskursverschiebung plausibel gemacht und so ermöglicht, das Opfer-Werden von Mitgliedern des ,Tätervolks' zu thematisieren, ohne einen automatischen Rekurs auf die Ursache (die deutsche Aggression) auszulösen.

\section{Die fiktionale Ebene des Zeugnisses}

Warum ein Sachbuch der Katastrophenerfahrung nicht gerecht werden kann, wird in den Worten der Protagonistin Tulla deutlich. Sie attestiert zwar dem Buch von Dobson, Miller und Payne, dass es mit ihren Erinnerungen übereinstimmt, will es aber nicht als authentische Darstellung gelten lassen, denn:

Das kommt nich von Härzen. (Grass 2002: 94)

Auch der Autor bekundet, dass er die literarische Darstellung Ereignisse jener Ereignisse, in denen die Deutschen zu Opfern wurden, für eine

20 Grass 2002: 211. Die Worte, die der Erzähler dem Opfer Brunies in den Mund legt, sind in ihrer symbolischen Dimension durchaus verfänglich: „Und auch mein Adoptivvater [..] hat Tulla von ihrer bösen Seite kennenlernen müssen. War bei ihr reiner Mutwille. Ging aber schlimm aus. Nach der Anzeige wurde Papa Brunies abgeholt ... Kam nach Stutthof ... Doch ist am Ende fast alles gut geworden.” (211). Der letzte Satz wird aus Opfersicht nur dann halbwegs erträglich, wenn man ihn auf die „Wandlung” Tullas nach 1945 bezieht. Es fällt auf, dass auch B. Schlinks Vorleser eine analoge Täterfigur in Nachkriegsverhältnissen konstruiert, deren Schuld durch klassische Markierungen von ,Schwäche' relativiert wird: Sie ist Frau, aus der Unterschicht, ungebildet, heimatlos, entwurzelt und alternd. Wo in früheren literarischen Auseinandersetzungen die Tätergeneration weniger milde beurteilt wird, sind die Repräsentanten männlich und agieren aus familiären, sozialen bzw. ökonomischen Machtpositionen, so z. B. bei Dürrenmatt, Koeppen, Böll, S. Lenz und Bachmann.. Vgl. GaLle 2002b. 
Notwendigkeit hält, ihre Unterlassung für einen Fehler, wenn nicht eine Schuld:

Niemals, sagt er [der „Alte”], hätte man über so viel Leid, nur weil die eigene Schuld übermächtig und bekennende Reue in all den Jahren vordringlich gewesen sei, schweigen, das gemiedene Thema den Rechtsgestrickten überlassen dürfen. Dieses Versäumnis sei bodenlos ... (Grass 2002: 99) gemacht wird. Man hört hier vielleicht ein Echo auf Adorno heraus, nämlich die ebenso so oft wie das, Lyrik-Verbot nach Auschwitz' zitierten Einschränkungen eben dieses Verdikts angesichts des „Leidens”: „Das Übermaß an realem Leiden duldet kein Vergessen [...]" (Adorno 1986, 11: 423) und vor allem die berühmte Passage aus der Negativen Dialektik: „Das perennnierende Leiden hat soviel Recht auf Ausdruck wie der Gemarterte zu brüllen [...]" (Adorno 1986, 6: 355 f.). Wie Adorno dem Gemarterten den Schrei, dem versehrten Menschen nach Auschwitz das gedenkende Gedicht, so möchte Grass offenbar den traumatisierten Flüchtlingen die Repräsentation ihrer Leiden zuzugestehen. Dass der Philosoph eine derartige Übertragung seiner Worte gutgeheißen hätte, darf man bezweifeln, denn für ihn stellte diese Lizenz schon die ,Ausnahmeregelung' dar, die nur sekundär Bestand haben kann vor der Frage, „ob nach Auschwitz noch sich leben lasse, ob vollends es dürfe, wer zufällig entrann und rechtens hätte umgebracht werden müssen" (ebd.). Im Vergleich zu den Überlebenden des industriellen Massenmordes, denen hier gerade noch das Recht auf Klage konzediert wird, hätte Adorno es wahrscheinlich als bodenlose Blasphemie empfunden, nun auch den Überlebenden des Täterkollektivs zuzugestehen, ihrer Opfer zu gedenken. Und doch erreicht die Formel des „perennierenden Leidens” bereits einen Grad an Universalität, der schwerlich die Frauen und Kinder der „Gustloff” ausschließen lässt. Auch wenn wir den gravierenden Unterschied zwischen vorsätzlichem Massenmord und fahrlässig fehlgeleiteter Kriegshandlung gegen einen zynischen Aggressor 
nicht vernachlässigen, erscheinen die getöteten Opfer aus der Distanz von über 50 Jahren gleichermaßen bedauernswert und in weitem Maße auch gleichermaßen ,unschuldig' an den Verursachungszusammenhängen, die zu Krieg und Massenmord geführt haben. Wenn schon das Subjekt der Geschichte sich aufgelöst hat, so lässt sich doch das Subjekt des Leidens an ihr trefflich für die ästhetische Darstellung einsetzen. Ohne dieses scheinbar passive Subjekt, das im Widerstehen und im Zeugnis-Ablegen dennoch agiert, sind auch die tatsächlichen Holocaust-Zeugnisse nicht denkbar (vgl. GALLE 2003).

Grass hat noch 1990 betont, dass Auschwitz nicht nur als Askesegebot von Anfang an über seiner lyrischen Produktion gestanden habe, sondern auch, dass er im Holocaust den historischen Einwand gegen die Vereinigung Deutschlands sehe. ${ }^{21}$ Dass man sich vor einem vereinigten Deutschland fürchten müsse, lässt sich auch aus Im Krebsgang entnehmen, doch hat sich die Strategie des Autors offenbar geändert: Da sich bestimmte Prozesse nicht aufhalten lassen, muss man ihnen korrigierend entgegenwirken. Bevor die „Rechtsgestrickten” die Erinnerung nationaler Opfer für sich vereinnahmen, hat ihnen die demokratische Mitte eine ungefährliche Gestalt zu geben.

Zugestimmt hätte Adorno vermutlich der Option für die literarische Form, denn sein Vergleich mit dem „Schrei des Gemarterten” bezog sich ja auf Gedichte, in denen die Verzweiflung von Überlebenden artikuliert ist. Charakteristisch für das Zeugnis des Überlebenden ist freilich seine doppelte Struktur: einerseits verzeichnet es das Erleben der Katastrophe durch den Berichtenden, andererseits enthält es den Verweis auf das paradoxe, unmögliche Zeugnis der Untergegangenen. Wenn man dieses Zeugnis als das eigentliche ansehen will, so ist es allenfalls in der Fiktion möglich, etwa in den Stimmen der Gedichte von Paul Celan und Nelly Sachs, bleibt dann aber wie alles Fiktionale hypothetisch und prekär. Die einzige Möglichkeit, das Zeugnis der Untergegangenen in einer nicht fiktionalen Aussage aufzuheben, liegt in der Referenz, die der Bericht des Überlebenden vermittelt, so z. B. Primo Levis berühmte Zeilen über

21 „Schreiben nach Auschwitz”. Grass 1997b 
Hurbinek und seine Ausführungen über die Figur des „Muselmann” (LEVI 1989: 190 ff.; LEVI 1990). ${ }^{22}$

Der Schrei der Untergegangenen ist es auch, der schon in den ersten Worten der Novelle beschworen wird und auf den der Erzähler immer wieder zurückkommt, andeutend in den ersten Zeilen:

Warum erst jetzt?" sagte jemand, der nicht ich bin. Weil Mutter mir immer wieder ... Weil ich wie damals, als der Schrei über dem Wasser lag, schreien wollte, aber nicht konnte ... Weil die Wahrheit nicht mehr als drei Zeilen ... warum erst jetzt. (Grass 2002: 7)

Dann im Hinblick auf die Traumatisierung Tullas:

„Da träum ech nich nur von, wie, als Schluß war, ain ainziger Schrei ieberm Wasser losjing.” (Grass 2002: 57)

Und schließlich auf dem Höhepunkt der Katastrophe und symbolisch parallel geführt mit dem Geburtsschrei des entronnenen Erzählers:

Doch soll ich, nach Mutters Erinnerung, mit meinem ersten Schrei jenen weithin tragenden und aus tausend Stimmen gemischten Schrei übertönt haben, diesen finalen Schrei, der von überall her kam: aus dem Inneren des absackenden Schiffsleibes, aus dem berstenden Promenadendeck, vom überspülten Sonnendeck, dem rasch schwindenden Heck und von der bewegten Wasserfläche aufsteigend, in der Tausende lebend oder tot in ihren Schwimmwesten hingen. Aus halbvollen und überfüllten Booten, von engbesetzten Flößen, die von Wellen gehoben wurden, in Wellentälern verschwanden, von überall her stieg gebündelt der Schrei auf und steigerte sich mit dem plötzlich einsetzenden, dann jäh erstickten Heulen der Schiffssirene zu grauenhafter Zweistimmigkeit. Ein nie gehörter, ein kollektiver Endschrei, von dem Mutter sagte und weiterhin sagen wird: „Son Jeschrai kriegste nich mehr raus aussem Jehör...” (Grass 2002: 146)

22 G. Agambens Theorie beruft sich zentral auf diese von Levi bewusst gemachte Struktur. S. AgAmBEN 1999. 
Am Anfang des Buches bekennt der Erzähler, er habe selbst schreien wollen, wie damals, als „der Schrei überm Wasser lag” - das ist hier noch ein wenig dunkel, auch die Anspielung auf Genesis 1.2, ${ }^{23}$ aber es lässt gleich zu Beginn die Ebenen ineinander gleiten: Autor, Erzähler, Zeugin, die Untergegangenen. Und mit diesen Ebenen verfließen hier die Motivationen, die raison d'être des Textes. Eine Motivation ist das unablässige Drängen der Mutter, eine weitere das eigene Bedürfnis nach einem Schrei, der den Geburtsschrei wiederholt und den Erzähler nachbarliche Verwandtschaft zu den Todesschreien der Opfer stellt. Zu vermuten ist, dass der Erzähler seinem Bedürfnis „zu schreien” mit dem Bericht nachgekommen ist. Da ihm Nüchternheit auferlegt ist und er seine Geburt nicht erfolgreich von dem Rettungsschiff auf die „Gustloff” phantasieren kann (vgl. Grass 2002: 146), ist es eher kein Schrei, sondern eben nur ein Bericht. Aber er enthält doch die Erinnerung der überlebenden Mutter, die sich über die Jahre zu einer zwanghaft wiederholten Rede über das Unglück verdünnt hat. Und er enthält den Schrei der Untergegangenen, der der Augenzeugin 'in den Leib gefahren ist'.

Die Erfahrung der Katastrophe hat Tulla innerlich und äußerlich sichtbar traumatisiert. ${ }^{24}$ Ihr Haar wurde in der Nacht des Unterganges schlohweiß ${ }^{25}$ und ihre Brüste verweigerten dem in dieser Nacht geborenen Erzähler ihren natürlichen Dienst, er muss von anderen Flüchtlingsfrauen gestillt werden. Vor allem aber teilt Tulla mit dem Holocaustzeugen die kognitive Verletzung: Sie trägt die traumatische Szene der ertrunkenen

23 „Und die Erde war wüst und leer, und es war finster auf der Tiefe; und der Geist Gottes schwebte auf dem Wasser." Die Bibel. Luther-Übersetzung. Stuttgart 1999.

24 Bezeichnenderweise hat Grass bereits in den Hundejahren eine Traumatisierung Tullas durch das Ertrinken eines Kindes geschildert: Der Badeunfall ihres kleinen, taubstummen Bruders Konrad, zu dessen Andenken der Enkel im Krebsgang auf denselben Namen getauft wird, lässt die halbwüchsige Tulla fünf Stunden lang schreien und sich dann sieben Tage beim Schäferhund Harras in der Hütte verkriechen. Vgl. Grass 1997: $182 \mathrm{ff}$.

25 „Das is bai de Jeburt von maim Sohn passiert. Ond zwar aufem Torpedoboot, was ons jerettet hat.” (Grass 2002: 55) und „Das is passiert, als ech all die Kinderchens koppunter jesehn hab...” (Grass 2002: 140) 
Kinder mit sich herum, die sie weder verarbeiten noch vergessen kann und die dazu drängt, in immer gleicher Weise erinnert und mitgeteilt zu werden.

Kam alles ins Rutschen. Kann man nich vergässen sowas. Das heert nie auf. Da träum ech nich nur von, wie als Schluß war, ain ainziger Schrei ieberm Wasser losjing. Ond all die Kinderchen zwischen die Eisschollen. (Grass 2002: 57)

Und, wie oben bereits zitiert:

„Son Jeschrai kriegste nich mehr raus aussem Jehör...” (Grass 2002: 146)

Der Zwang, das unverarbeitet gebliebene Erleben zu verbalisieren, überträgt sich von Tulla auf den Sohn und auf den Enkel. Der Erzähler bemerkt mehrfach explizit, er würde seine Geschichte nicht aus freien Stücken festhalten. Was ihn zwingt ist zum einen der „Alte”, der seinerseits bekundet, das Thema sei ihm „auferlegt” worden, nachdem er die Hundejahre abgeschlossen habe und damit quasi zum zeitkritischen Chronisten jener durch Nazis und Krieg gezeichneten Generation geworden war. Zum anderen ist es aber eigentlich seine Mutter, die von ihm fordert, er müsse „die Geschichte” aufschreiben und der Nachwelt überliefern.

Ech leb nur noch dafier, daß main Sohn aines Tages mecht Zeugnis ablegen. (Grass 2002: 19)

Nicht etwa, weil mir der Alte im Nacken sitzt, eher weil Mutter niemals lockergelassen hat. Schon in Schwerin, wo ich, wenn irgendwas eingeweiht wurde, mit Halstuch und im Blauhemd rumhampeln mußte, hat sie mich gelöchert: „Wie aisig die See jewesen is und wie die Kinderchen alle koppunter. Das mußte aufschraiben. Biste ons schuldig als glicklich Ieberlebender. Wer ech dir aines Tages erzählen, klitzeklain, ond denn schraibste auf..." (GRAss 2002: 31)

Die Gewalt, die der Gustloff-Überlebenden zugeschrieben wird, ist analog jenem Drang, der den Zeugen dazu treibt, Zeugnis abzulegen. Primo Levi hat - wie andere Auschwitz-Häftlinge vielfach auf diesen Impuls hingewiesen, am eindrücklichsten in dem von Coleridge entlehnten Bild im Motto zu den Untergegangenen und Geretteten. 
Since then, at an uncertain hour, That agony returns:

And till my ghastly tale is told This heart within me burns. ${ }^{26}$

In Die große Reise von Jorge Semprun wird an der Struktur des Textes selbst nachvollziehbar, wie sich Erinnerung aufdrängt und zur Mitteilung drängt:

Und ich habe wirklich vergessen, ich habe alles vergessen, alles ist nur noch Erinnerung für mich. Ich kann diese Geschichte der jüdischen Kinder aus Polen jetzt erzählen, nicht wie eine Geschichte, die mir ganz persönlich zugestoßen ist, sondern die vor allem diesen jüdischen Kindern aus Polen zugestoßen ist. Mehr noch, es ist mir nach diesen langen Jahren freiwilligen Vergessens nicht nur möglich, sie zu erzählen, ich muß es tun. Ich muß sie nennen, nicht in meinem eigenen Namen, sondern im Namen der Dinge, die geschehen sind. Ich muß die Geschichte der jüdischen Kinder im Namen der jüdischen Kinder selber erzählen. Ich muß die Geschichte ihres Todes auf der großen Straße, die zum Eingang des Lagers führte, unter den steinernen Blicken der Naziadler, unter dem Lachen der SS-Männer erzählen, im Namen dieses Todes selber.” (SEMPRun 1981: 166)

26 Levi 1990: 5. Über die Persistenz der traumatischen Situation bemerkt Jean Améry: „Es war für einmal vorbei. Es ist noch immer nicht vorbei. Ich baumele noch immer, zweiundzwanzig Jahre danach, an ausgerenkten Armen über dem Boden, keuche und bezichtige mich. Da gibt es kein, Verdrängen'. Verdrängt man denn ein Feuermal?” AMÉRY 1988: 54. Ruth Klüger: „,[..] wenn es auch stimmt, daß wir, anders als ihr, unser Leben lang etwas Mitgeschlepptes von diesem Ort durchspielen oder -spielten, so wie ich auf die Rampe falle, wenn ich schlecht schlafe, wenn ich aus der Narkose erwache, wenn ich mich in Lebensgefahr befinde. (Ein sehr schwacher Wiederholungszwang übrigens, verglichen mit anderen.)" KLÜGER 1997: 141.

Auch der Autor selbst hat angegeben, ihm sei „die verlorene Heimat zum andauernden Anlaß für zwanghaftes Erinnern, das heißt für das Schreiben aus Obsession geworden.” Grass 2001. 
Wie bei Semprun geht es auch bei Tulla letztlich nicht um das Leiden des oder der Überlebenden, sondern um das in ihrer traumatischen Erinnerung eingeschriebene Leiden der Untergegangenen. Sie selbst verweist immer wieder auf jenen unartikulierten Schrei des Kollektivs, für das es keine Rettung gegeben hat. Auch diese Struktur des Zeugnisse, das nie das eigentliche Zeugnis ist, sondern nur stellvertretend für das unmögliche Zeugnis jener einsteht, die "das Haupt der Medusa erblickt” haben. ${ }^{27}$ Der Schrei, der in der Nacht des 30. Januar über der Ostsee verhallte, war Ausdruck jenes ,Übermaßes an Leiden', das nur durch die Vermittlung einer menschlichen Stimme Eingang in unsere Erfahrung finden kann.

In diesem Fall ist die Stimme freilich die einer fiktionalen Figur, deren Individualität durch dialektales Sprechen gegen den blassen Stil des Erzählers abgehoben wird. Glaubwürdig wird sie jedoch vor allem durch die enge Verzahnung mit den Fakten und ihren ambivalenten und symbolischen Charakter, der auf die „Danziger Trilogie” zurückgeht. Seit Hundejahre gehören Tulla Pokriefke und Walter Matern gewissermaßen zum mythologischen Personal der Bundesrepublik - wie Franz Biberkopf und Familie Buddenbrook zu dem der Weimarer Republik bzw. des Kaiserreichs.

Ein authentisches Zeugnis, wie es die Bücher Primo Levis, Jean Amérys, Ruth Klügers und Jorge Sempruns darstellen, kann freilich durch diese fiktive Person nicht gewährt werden, denn nur die Identität von Autor, Erzähler und Protagonist (im Sinne von Philippe Lejeunes Autobiographie-Pakt) kann dem Leser gegenüber dafür bürgen, dass die geschilderte Erfahrung so erinnert wird. Grass scheint dies aber nicht zu beunruhigen, denn er kann immerhin Zeitzeugenschaft für sich beanspruchen, wenn auch keine Augenzeugenschaft. In diesem Dreieck von Faktengrundlage, Zeitgenossenschaft und fingiertem Augenzeugenbericht hat der Erzähler doch einen relativ sicheren Stand, wenn er das Geschehen um die Gustloff dem kollektiven Gedenken empfehlen will. Wenngleich die vom kollektiven Gedächtnis überlieferten Inhalte - im Unterschied zur Geschichtswissenschaft - stets eine gewisse Nähe zur Struktur des Mythos behalten, so lässt sich doch konstatieren, dass sie in zunehmendem Maße an die rationale Aufarbeitung zurückgebunden werden. Anders als im

27 Levi 1990: 83; vgl. auch Agamben 1999. 
19. Jahrhundert sind es nicht mehr die Helden mittelalterlicher Epen oder nigromantischer Renaissance-Legenden, die zur Identifikation herhalten. Die Konjunktur der Zeugentexte mag daher gerade auch mit ihrer Zwischenstellung zwischen Literatur und Geschichtsschreibung zu tun haben.

Man sollte aber auch anmerken, dass die Lagererfahrung der Zeugentexte und der Schiffsuntergang nicht auf derselben Stufe des Grauens stehen. Um dies zu belegen, würde es ausreichen, das obige Zitat von Jorge Semprun fortzuführen. Es würde schnell klar werden, dass die Realität des Lagers eine bis dahin völlig unvorstellbare Dimension dessen darstellte, was Menschen Menschen antun können. Die Zeugen der Shoa sehen sich daher vor der Aufgabe, die Häftlingsexistenz überhaupt erst zu beschreiben, angemessene Narrative für sie zu finden, und bleiben doch weit davon entfernt, sie zu verstehen. So kommt ihnen prinzipiell eine enormer Wert für den intellektuellen Zugriff auf diese scheinbar so zentrale soziale Konfiguration der Moderne zu, während die Pathos-Wirkung auf das Publikum lediglich sekundär bleibt. Nun kann man zwar auch für die Versenkung der „Gustloff” in Anspruch nehmen, dass sie ein „,man-madedesaster" ist, Teil der inhumanen, technisierten Kriegsführung, bei der durch einen Knopfdruck Tausende von unschuldigen Zivilisten ohne jeden strategischen Nutzen geopfert werden. Aber für die Erfahrung der Katastrophe unterscheidet sich doch kaum von der anderer Seeunglücke, die auf Passagierschiffen durch ganz natürliche Ursachen - Eisberge etwa - ausgelöst werden. Die Inhumanität des U-Boot-Kommandanten Marinesko, der die Torpedos in Gang setzen lässt und die Inhumanität des Auschwitzkommandanten Höß, der über die Massentötungsmaschinerie gebietet, mögen sich aus vergleichbaren psychischen und sozialen Konfigurationen herleiten, aber sie wären moralisch und juristisch nicht mit dem gleichen Maß zu messen, ganz abgesehen von der Frage der historischen Priorität.

Aber es soll auch nicht unterstellt werden, Grass habe es bei der Anlehnung an das Zeugnismuster auf irgendeine Gleichsetzung von Shoa und den Leiden Deutscher gegen Ende des Krieges abgesehen. Ihm geht es nicht um die Revision des Verhältnisses von verursachter und erlittener Katastrophe, sondern um eine Revision des Verhältnisses von Schuld und Leiden in der, internen Perspektive'. Man mag anzweifeln, dass es eine solche 
gewissermaßen nationale Perspektive im 3. Jahrtausend in Mitteleuropa überhaupt noch geben kann oder sollte. In sehr vielen Bereichen ist es ganz sicher sinnvoll diese Perspektive ganz zu verlassen. Aber ebenso sicher scheint es, dass die Deutschen - hier ist der Verfasser symptomatischerweise versucht, das ,wir” einzusetzen - eine eigene Sicht der Vergangenheit haben und haben müssen, wenn es um die Verantwortung für Auschwitz geht. ${ }^{28}$ In dem Maße, in dem sie zu akzeptieren haben, dass diese Verantwortung in allererster Linie von einer Generation getragen wird, deren Handlungen und Haltungen sie - wie vermittelt immer - beerbt haben, in dem Maße sind sie auch dafür zuständig, der Opfer zu gedenken, die in ihrer eigenen Mitte gebracht wurden, auch wenn diese Opfer unauflöslich an die historische Schuld des Kollektivs gekettet bleiben.

Sofern diese ,Innenperspektive' des kollektiven Gedächtnisses eine Realität ist, sollte es auch sinnvoll sein, sie zwischen Schuld, Verdienst und Erleiden auszubalancieren. Genau darauf zielt die Novelle, wenn sie die Tabuisierung der deutschen Opfer mit neonazistischer Gewalt in der dritten Generation in Zusammenhang bringt.

\section{Die fiktionale Ebene der Propaganda}

Die im Chatroom ausgetragene Debatte über die tatsächlichen Ereignisse um die Gustloff und ihre Bedeutung für die Gegenwart verläuft in einer Polarisierung, welche die ideologisierten Identitäten der dreißiger Jahre wiederholt. Dem nicht prinzipiell bösartigen Konny erscheint die öffentliche Diskussion, in die er hineingeboren wird, zu einseitig und zu ausschließlich auf die jüdischen Opfer fixiert, weiß er doch aus der kommunikativen Erinnerung der Familiengeschichte, dass sich damit nicht alle wichtigen Erfahrungen des Krieges abdecken lassen. Da seine Initiativen zur Korrektur dieses Bildes auf institutionellen Widerstand stoßen, verhärtet

28 Vgl. Dan Diner: „Kein deutscher Bürger nicht-ethnisch deutscher Herkunft kann vernünftigerweise dazu verpflichtet werden, an einem kollektiven Gedächtnis zu partizipieren, das im Kontext einer historischen Erinnerung eingebunden ist, die ihn gerechterweise zu nichts verpflichtet." DINER 1995: 119. 
sich seine Position, und er schließt aus der öffentlichen Verdrängung des Opferaspekts, dass der vom gleichen Tabu betroffene Aspekt der nazistischen bzw. deutschen Schuld ebenfalls einer Revision bedarf, was scheinbar durch Elemente wie die Kraft-durch-Freude-Organisation und Tullas diesbezügliche Erinnerungen bestätigt wird. So gerät er auf die Fahrbahn des Rechtsextremismus und findet schließlich einen gleichaltrigen Gegner, der ebenso hartnäckig die Gegenseite einzunehmen bereit ist.

Die Virtualität des neuen Mediums ermöglicht beiden, ihre Äußerungen losgelöst und ungemildert von realen sozialen Kontexten weiter zu radikalisieren: Beide steigern sich in ihren Streit und die Rollen hinein, ohne dass die Eltern und das Umfeld etwas davon ahnen und moderierend eingreifen könnten. Das Medium begünstigt nicht nur die Verselbständigung extremer Positionen, es entbehrt auch der Kontrollmechanismen, die eine Aussage - wie der Markt der Druckwaren - Prüfungen unterziehen, bevor sie der Öffentlichkeit zugänglich wird. Ob das neue Medium tatsächlich die von Grass befürchteten Erscheinungen fördert, ist sicher fraglich. Im Plot der Geschichte erscheint die Darstellung einigermaßen plausibel und verhilft Grass außerdem zu einer markanteren Scheidung des von ihm selbst betriebenen Literaturhandwerks und den ,im luftleeren Raum' agierenden Ideologen. Beide verfechten eine exklusive Erinnerungspolitik im Interesse jeweils einer Partei und rechtfertigen deren Gewaltakte: Konny im Sinne der Nazis, David im Sinne der Juden und der Kriegsgegner Nazideutschlands (Grass 2002: 149 f.). Sie setzen damit auch die in den dreißiger Jahren bereits publizierten parteilichen Publikationen zu Frankfurter und Gustloff fort: Auch Diewerges und Ludwigs ${ }^{29}$ Bücher interpretieren das historische Geschehen mit antagonistischen ideologischen Formeln. Wie die ältere

29 Emil Ludwigs Publikation (1936 und, erweitert, 1945) weist sich schon in Titel („David und Goliath”) und Vorwort als eine Verteidigungsschrift für den jüdischen Attentäters aus; der Autor versucht mit allen Mitteln psychologischer Einfühlung und politischer Rechtfertigung, den Leser von der „Unschuld” Frankfurters zu überzeugen. Übrigens scheint sich Adornos vernichtendes Wort zur „biographischen Schundliteratur” (ADORNo 1986: 163) auf Ludwig zu beziehen, der als ,Erfinder' und Hauptvertreter der „historischen Biographie” in der Zwischenkriegszeit neben Stefan Zweig zu den erfolgreichsten Autoren überhaupt zählte. 
Literatur sich auf die „höhere Wahrheit” dichterischer Imagination verlassen hat, so glauben auch die beiden Internetbenutzer, ihre subjektiven Phantasien könnten für die Erkenntnis der Vergangenheit herhalten:

Mein Sohn gab vor, genau zu wissen, daß das Ortungsgerät des Hipper-Begleitschiffes nicht nur U-Bootgefahr ausgemacht habe [...]. Woraufhin David, als wäre er unter Wasser dabeigewesen, bezeugte, wie unbewegt, ohne das Sehrohr auszufahren, das erfolgreiche sowjetische U-Boot sich verhalten und keinen einzigen Torpedo abgeschossen habe [...]. Und nun begann die im Internet mögliche Freizügigkeit der totalen Kommunikation. [...] Ein jeder sollte nun wissen und beurteilen, was am 30. Januar 1945 auf de Stolpebank geschehen war. (GRAss 2002: 148 f.)

In welcher Weise sich die Tätigkeit des Erzählers Paul von dieser Praxis auktorialen Erzählens unterscheiden will, wurde bereits angesprochen. Dass der Text als Ganzes - gestützt auf eine andere, verantwortlichere Form von historischer Darstellung - wiederum ein Gedächtnis stiften soll, durch das die Täter nicht gerechtfertigt, aber auch keine Opfer ausgeschlossen werden, wird im Weiteren noch genauer ausgeführt. Doch zunächst noch in aller Kürze zur Rolle der dritten Generation in der Novelle.

Wie schon die Nazipropaganda das Mordopfer Wilhelm Gustloff zum Märtyrer verklärte und zur Rechtfertigung weiterer Aggressionen instrumentalisierte, so benutzt Konny das Unglück seiner Großmutter, um ,Vergeltungsmaßnahmen' zu legitimieren. Die Identifikation mit dem Opfer dient hier - wie meist in neonazistischen Konstrukten - zur Verschleierung der eigentlichen Identifikation mit dem Aggressor: Tulla steht eben nicht nur für die Niederlage, sondern auch für Macht und ,Glanz' des dritten Reiches. (Diese Ambivalenz deutet sich z. B. symbolisch an, wenn sie zum Prozess ihres Täter-Enkels den Fuchspelz umlegt, der gegen alle Wahrscheinlichkeit aus der Danziger Zeit herübergerettet wurde.) Für den adoleszenten Konny hat dieses Modell mehr zu bieten als das angepasste und handlungsscheue Verhalten des Vaters und seiner Generation, die, sich selbst zu Opfern ihrer Tätereltern stilisierend, es ablehnen, selbst zu handeln und schuldig zu werden. Dass diese Strategie die von ihr beanspruchte höhere Moral nicht einlösen kann, wird von Konny leicht durchschaut. 
Obschon der Rekurs auf die Märtyrer ein (mitunter sogar unbewusster) Vorwand ist, leuchtet die Verknüpfung von Erinnerungsverbot und Fehlentwicklung im Handlungszusammenhang ein. Tabus begünstigen in letzter Konsequenz mythische Überhöhungen, Heldenerzählungen. Das Tabu war zwar durchaus nicht, irrational', da es in einer konkreten Phase der Nachkriegsentwicklung die rückhaltlose Anerkennung der Verantwortung ermöglichte, doch nachdem diese erfolgreich etabliert wurde, verdient das Tabu getrost als solches bekannt und aufgehoben werden. Das kollektive Gedächtnis der Deutschen hat sich nach '45 in sukzessiven Wellen immer weiter der historischen Aufklärung geöffnet. In diesem Prozess ist die eigene Perspektive immer wieder an der Sichtweise der anderen beteiligten Nationen und der diversen Opfergruppen ${ }^{30}$ korrigiert worden. Einzelne Maßnahmen der Alliierten gegen die deutsche Zivilbevölkerung in der Endphase des Krieges sind allerdings Bereiche, die noch weiterer Aufklärung und Diskussion bedürfen, damit es nicht den „Rechtsgestrickten” gelingt, das Vakuum mit verzerrenden Darstellungen zu füllen. Ein solches Unternehmen ist das Buch Grass' als ganzes: Es sichtet die Faktenlage und spekuliert über den Missbrauch, den man mit ihr treiben könnte, sofern sich die Öffentlichkeit nicht auf klarere Kenntnisse stützen kann.

Die beiden Repräsentanten der dritten Generation sind allerdings letztlich nicht sonderlich überzeugend geraten. Die antifaschistische Position und jüdische Identität bei „David” wird wohl eher dem linken Hauptstrom der zweiten Generation gerecht. Andererseits kann nicht geleugnet werden, dass es unter der dritten Generation eine beunruhigende Neonazi-Szene gibt, die seit der Vereinigung durch eine Serie von blutigen Aktionen auf sich aufmerksam gemacht hat, so dass der Mord Konnys an David zumindest auf symbolischer Ebene - nicht absurd ist, auch wenn er in der konkreten Motivierung künstlich bleibt und die Erzählung über dieses Opfer ebenso auffällig desinteressiert hinweggeht wie dessen fiktive Eltern. In der Realität hingegen ist für die Enkel im Allgemeinen eigentlich eine

30 Dass diese Opfer zu einem nicht geringen Teil Deutsche waren, bevor sie in die Mühlen der nazistischen Nomenklaturen gerieten, braucht nicht betont zu werden. 
distanziertere und neutralere Haltung zu den Großeltern typisch, während Konny und „David” in der Novelle geradezu als Wiedergänger der Täter/ Opfergeneration agieren.

Jörn Rüsen hat die Generationenfolge im Hinblick auf das 3. Reich dahingehend analysiert, dass es nach Verdrängung in der ersten und Identifikation mit den Opfern in der zweiten nun zu einer Reintegration genealogischer Kontinuität komme und die Generation der Enkel die Handlungen ihrer Großeltern beurteilen könne, ohne sich selbst als deren Opfer zu stilisieren aber auch, ohne sich mit ihnen in ihrer Eigenschaft als Täter zu identifizieren. ${ }^{31}$ $\mathrm{Zu}$ dieser ,Normalisierung' im Generationenverhältnis gehört einerseits das Anerkennen der Bedingtheit individuellen Handelns in der Kontingenz biographischer Situationen, und andererseits das Anerkennen von Leiden, das von den Alliierten verursacht wurde. Beides wird von Grass' Buch, auch wenn er selbst ein Angehöriger der ersten Generation ist, beispielhaft in Szene gesetzt und historisch hinreichend unterfüttert.

\section{Kollektive Erinnerung an den Krieg nach 1990}

Wenn der Titel der Novelle - ein metaphorischer Verweis auf die Erinnerungsarbeit des Erzählers - zunächst dunkel bleiben mag, so ist bereits das Motto in memoriam ein klarer Hinweis auf die Absicht des Autors, einen Beitrag zum kollektiven Gedächtnis zu leisten. Nicht dem Andenken einer einzelnen Person ist der Text gewidmet, sondern dem Eingedenken an sich, dem Gedächtnis als Institution. Was vor dieser Instanz erinnert werden soll, wird sich im Verlauf der Lektüre entfalten. Wenn ein Nobelpreisträger, der für jedes seiner Bücher mit Startauflagen rechnen kann, von denen andere Autoren nur träumen, sich mit solchen Worten an ein Publikum wendet, das in den vergangenen Jahrzehnten immense Anstrengungen auf die Neubildung einer Gedenk-Kultur verwendet hat, erhebt er mit dem Motto keinen geringeren Anspruch, als dass die von ihm reklamierten Geschehnisse vor diesem Forum verhandelt und mittels dieses Textes in den Kanon nationalen Gedenkens eingeschlossen werden wollen. Und - nicht erst im Zeichen der Globalisierung

31 Vgl. den Aufsatz „Holocaust-Erinnerung und deutsche Identität” in: RüsEN 2001: 278-299. 
- präsentiert er diesen deutschen Untergang vielleicht sogar „der ganzen Welt”, wie es Tulla von ihrem Sohn, dem Erzähler, einmal erwartet hatte (GRASs 2002: 32). Da seit den Ereignissen bereits zwei Generationen vergangen sind, würden sie-im Zuge des biologischen Prozesses - allmählich aus dem kommunikativen Gedächtnis verschwinden und zu bloßem, historisch dokumentiertem Material werden, nähme die Gemeinschaft der Nachgeborenen sie nicht in den Fundus jener Erfahrungen auf, die ihren Bestand gewährleisten und ihr Handeln orientieren. ${ }^{32}$ Die kollektive Erinnerung der Deutschen hat in den Jahren seit dem Krieg bekanntlich eine radikale Wandlung durchgemacht und die Verantwortung für Genozid und Vernichtungskrieg in ihre Identität integrieren müssen. Während dieses Prozesses wurden die Erinnerungen von solchen Gruppen aus dem öffentlichen Diskurs ausgeblendet, die in der Spätphase des Krieges und der Nachkriegszeit selbst Opfer von Unrecht und Gewalt wurden, in erster Linie die Bewohner der ausgebombten Städte, Flüchtlinge aus dem Osten, Vertriebene und im Zuge der Besetzung vergewaltigte Frauen. Es galt damals, im Interesse einer politischen und moralischen Rehabilitation der Bundesrepublik, jene Stimmen zu verdrängen, die auf eine revanchistische Revision des status quo drängten oder auch nur die Eindeutigkeit der initialen deutschen Verantwortung in Frage zu stellen durch ein relativierendes Aufrechnen von Kriegsverbrechen und unschuldigen Opfern auf beiden Seiten. Der namentlich nach der Vereinigung und der Anerkennung der polnischen Grenzen zustande gekommene politische und historische Abstand ermöglicht nunmehr eine Neukonfiguration der nationalen Identität, in die das Gedächtnis an Leiden der Bevölkerung aufgenommen werden kann, ohne problematische politische Folgen zu zeitigen. ${ }^{33}$

32 Vgl Assmann 2002: „Sinn, wie ihn Kulturen produzieren, um soziales Handeln in einem Horizont gemeinsamer Erfahrung und Erwartung zu fundieren [...] wird typischerweise aus der gemeinsamen Geschichte, bes. aus kollektiven Leiderfahrungen gewonnen [...]." Assmann 2002: 100. Zu kommunikativem und kulturellem Gedächtnis vgl. Assmann 1997: 48 ff.

33 Auch Aleida Assmann sieht in einer Rückkehr der Erinnerungen zum gegenwärtigen politischen Zeitpunkt keine Gefahr mehr, nachdem sich der Holocaust als zentrales Paradigma nationalen Gedenkens etabliert hat und die Vertriebenenverbände keinen gesellschaftlichen Machtfaktor mehr darstellen. AsSMANn 2004. 
A. Huyssen hat davon gesprochen, der Holocaust sei zum ,globalen Modellfall für die Verarbeitung nationaler Traumata" geworden (HuYsSEN 2004). Dass auch die Deutschen das Modell benutzen könnten, um der Opfer zu gedenken, die von alliierter Seite verursacht wurden, ist bislang von der Schuldfrage verdeckt worden. Eine Rekonfiguration der kollektiven Memoria liegt jedoch durchaus nahe und erscheint nicht nur als protokollarische Geste gegenüber denen, die auf deutscher Seite zu den ,eigentlichen Verlierern des Krieges' gehören, weil größere Verletzungen der Identität - vor allem kollektive Katastrophen - ihre untergründigen Spuren hinterlassen und nach einer Latenzzeit wie das individuelle Verdrängte wiederkehren könnten. Dies wird zumindest von der Handlung der Novelle nahegelegt, welche die Gewalt in der dritten Generation wieder aufleben lässt. Es soll hier nicht entschieden werden, ob auf der symbolischen, Ebene die Verwandlung des verdrängten Traumas ${ }^{34}$ in manifeste Aggression von den entsprechenden psychoanalytischen Theorien gedeckt ist, das heißt, ob es angemessen ist, hier von kollektiver Verdrängung eines (Verletzungs-)Traumas und der Wiederkehr des Verdrängten zu sprechen. ${ }^{35}$ Rechtsradikal motivierte Gewalt scheint im Gegenteil eher mit

34 Für die Ebene der fiktionalen Handlung greift die Trauma-Theorie ohnehin nicht, denn Tulla Pokriefke hat ihre Erfahrung ja nicht verdrängt, sondern fordert im Gegenteil immer wieder, dass sie öffentlich mitgeteilt werde. Familiengeschichtlich wird also nicht ein Geheimnis durch Beschweigen an Kinder und Kindeskinder weitergereicht. Auf der symbolischen Ebene dagegen, findet in der Tat eine gesellschaftliche Verdrängung der Opfer statt. Da aber gleichzeitig derselbe Ereigniskomplex mit der durchaus nicht verdrängten Schuld der Deutschen verknüpft wird, bleibt fraglich, ob hier die herkömmliche psychoanalytische Herangehensweise und Terminologie überhaupt angemessen ist.

35 Historisch war es ja das gerade nicht verdrängte Trauma des 1. Weltkrieges, das mit zum immensen Gewaltausbruch der Nazizeit geführt hat. Dagegen scheinen die unter Traumata leidenden Holocaustopfer andererseits nicht zur Gewaltanwendung zu neigen. Wie immer man die Psychoanalyse als Instrument zum Verständnis individueller Psyche beurteilen mag, die Übertragung auf kollektive Prozesse scheint wenig gesichert. Im Hinblick auf die deutsche Geschichte werden psychoanalytische Kategorien z.B. von Chris Lorenz gebraucht. LoRENz 2000 u. 2002. Zur psychoanalytischen Beschreibung der Übertragung traumatischer Erfahrungen in der zweiten Generation von Opfern u. Tätern vgl. u.a. BOHLEBER 1998. 
der Abwehr von Schuld ${ }^{36}$ zu tun zu haben, Motive wie die im Krebsgang geschilderte Gerechtigkeitsliebe des Enkels sind dann - wie bereits erwähnt - vorgeschützt. Doch findet über den Mord und seine strafrechtliche Sühne nun auch die zweite Generation ein neues Verhältnis sowohl zu den Eltern wie zu den Kindern. ${ }^{37}$

Man kann in der Rückkehr des Leidens deutscher Opfer aber auch eine Verschiebung aus dem (kommunikativen) Familiengedächtnis ins

36 Vgl. DiNer 1995:118.

37 Vgl. hierzu Rüsen, LoRENZ und Schlink. Schlink macht deutlich, dass der wesentliche Unterschied zwischen 2. und 3. Generation darin besteht, dass die Kinder der verantwortlichen Generation die „Kollektivschuld” erben, weil die soziale Verstrickung in ihren Ausmaßen eine Ausgrenzung und Bestrafung der eigentlich Schuldigen letztlich unmöglich machte und dadurch im Nachkrieg die Nichtausgrenzung faktisch zur Solidarität und diese zur Mitverantwortung wurde. Vererbte Kollektivschuld beruht demzufolge nicht auf dem „Blut” oder einem „Fluch”, sondern auf einem bestimmten Verhalten gegenüber den Schuldigen. „Das bedeutet, dass sich die Verstrickung bei der dritten Generation löst. Die dritte Generation steht nicht mehr vor der Alternative, die Täter auszustoBen oder in der Gemeinschaft zu halten; sie kann sich dadurch, dass sie die Täter nicht ausstößt, nicht in deren Schuld verstricken.” Vgl. SCHLINK 2002: 97 ff. Da die zweite Generation in diese Solidarität mehr oder weniger unbewusst hineingewachsen ist, wurde sie von den Dimensionen dieses Erbes erst zu einem Zeitpunkt erschüttert, als es gar nicht mehr zurückzuweisen war, es sei denn um den Preis des radikalen Bruchs mit der gesamten Generation, mit der Aufkündigung des stillschweigenden Einverständnisses zwischen den Generationen, das in allen Gesellschaften für Kontinuität sorgt. Rüsen: „Man kann vom Zerbrechen einer intergenerationellen Erinnerungsgemeinschaft sprechen. Ein solches Zerbrechen stellt eine schwere Störung von historischer Identität dar." (RüsEN 2001: 289) Dass dieser Bruch dennoch von einem entscheidenden Teil der 2. Generation vollzogen wurde, führte zu den spezifischen Verhältnissen in Deutschland, für die Paul Pokriefke als Symbolfigur herhalten kann: Er lebt in einem anderen Staat als seine Mutter, stellt ihre Angaben über seine Geburt und Herkunft in Zweifel und entzieht systematisch sich ihren Ansprüchen, bis ihn die Handlungsweise seines Sohnes zu einer Wendung zwingt, während seine Frau, ebenfalls eine symbolische Repräsentantin der Täterkinder auch weiterhin die Anerkennung der Eltern und ihres Erbes an Verantwortung verweigert und sich in einer Scheinrealität für moralisch integer hält. 
(kulturelle) nationale Gedächtnis sehen, wie Aleida Assmann dies getan hat. Sechzig Jahre nach den Ereignissen stehen die Erinnerungen momentan an der Schwelle eines sich auflösenden Erfahrungsgedächtnisses, das in absehbarer Zeit vollständig verschwunden sein wird. An dieser Schwelle meldet es sich noch einmal mit neuer Emphase zurück. Die Voraussetzung dafür ist, dass auf die „Erfahrungsgeneration” eine „Bekenntnisgeneration” folgt, die diese Erinnerung zu ihrer eigenen macht und Anstrengungen unternimmt, das Gedächtnis ihrer Eltern vor dem Verschwinden und Vergessen zu bewahren. Diese Umorientierung vollzieht sich insbesondere durch einen Generationenwechsel von der zweiten zur dritten Generation, die neue Erinnerungsprofile und Identitäts-Bedürfnisse entwickelt. Nach Konflikt und Bruch stehen nun wieder Harmonisierung und Kontinuität im Vordergrund. (Assmann 2004)

Der Erfahrungsgeneration angehörend hat der Autor Grass es nicht dem Zufall überlassen, dass sich die Enkel des Stoffes annehmen, und das Bekenntnis den fiktionalen Gestalten seiner Novelle überantwortet. Es ist jedoch erkennbar, dass in den letzten Jahren die Zahl der Texte stetig gewachsen ist, die sich mit der Thematik befassen. ${ }^{38}$

Fragt man abschließend noch einmal nach der Leistung der hier analysierten Struktur der Novelle im Vergleich zu authentischen Zeugnissen, historiographischer Dokumentation und ,reiner" Fiktion, so wird klar, dass keine dieser Gattungen in ihrer Reinform es mit der Novelle aufnehmen kann.

Eine lediglich imaginierte Geschichte über Flucht und Vertreibung der Deutschen nach dem Krieg kann nur dann zu einer erfolgreichen Überschreibung der kollektiven Erinnerung beitragen, wenn sie auf breiter, gesicherter Faktenlage einen Erklärungsaspekt beiträgt, der sich der psychologischen Zeichnung der spezifischen Charaktere vor dem Hintergrund ihrer Verhältnisse und in ihrer Entwicklung verdankt. In diesem Sinne wirft etwa das Personal der Danziger Trilogie über die gesellschaftsund wirtschaftsgeschichtlichen, die politischen und verfassungsrechtlichen

38 Vgl. etwa Tanja Dückers Buch zur „Gustloff”, Götz Friedrichs Der Brand, Kempowskis Echolot-Projekt (insbesondere Fuga furiosa und Der Rote Hahn) und Götz Berganders Dresden im Luftkrieg. 
Studien hinaus Licht auf Diktatur und Nachkriegszeit. Die Erfahrung der Opfer war zwar in den 50er Jahren im kommunikativen Gedächtnis präsent und wurde auch wissenschaftlich aufgearbeitet, es drohte jedoch gegenüber der unabweisbaren Anerkennung der deutschen Täterschaft zur ablenkenden Scheinerinnerung zu mutieren; ${ }^{39}$ die Opfererfahrung bedarf also von neuem einer gesicherten Faktenunterlage, auf der sich die Fiktion überhaupt erst entfalten ließe.

Die Dokumentation der Ereignisse vom Untergang der „Gustloff”, die Gründe für den Abschuss, die Zahlen der Ertrunkenen, das Schicksal der Geretteten usw. lagen auch vor Grass' Erzählung vor, sind dieser aber von vornherein an emotionaler Wirksamkeit unterlegen, welche die Einbindung in eine lebendige Biographie gewährt. Diese triviale Qualität teilt sie mit einer fiktionalen Inszenierung im Stile des Titanic-Filmes. Die Novelle hat dem Medienspektakel jedoch voraus, dass ihre Konstruktion nicht eine vergangene Realität eindimensional vorspiegelt, sondern die Ereignisse aus der Perspektive der Gegenwart heraus so darstellt, dass sie in sich gebrochen ist durch die unterschiedlichen Standpunkte der Augenzeugin, des recherchierenden Erzählers und des politisch motivierten Enkels. Geschichtliche Realität erscheint dadurch von vornherein als eine solche, die im Horizont der Zeit um die Jahrtausendwende formuliert wird und die vorangegangene Erfahrungen aufgenommen hat. Zwar wird auch die Titanic-Fiktion aus dem gegenwärtigen Horizont heraus konstruiert, doch sind die Prozeduren, mit denen das Vergangene perspektiviert wurde, ausgeblendet, während die Novelle diese explizit mitreflektiert. Der Leser hat teil an den Textkonstitutions- und Integrationsprozessen, die der Erzähler mit den Erinnerungen seiner Mutter, den Dokumenten, seinen eigenen Phantasien und den revisionistischen Versionen seines Sohnes vornimmt.

Auch ein authentischer Augenzeuge ‘deutschen Leidens' könnte die Emotionen des Lesers ansprechen. Sein Bericht wäre jedoch in keinem Fall

39 Die Präsenz von Dokumenten in den Archiven, A. Assmanns „Speichergedächtnis”, besagt nichts über deren Wirksamkeit für das kollektive Gedächtnis. Dies kann zwar mit Rückgriff auf die Speicher neu formiert werden, funktioniert aber nur mittels Ausblendung des gesamten Speichermaterials und Beschränkung auf das Kanonische. 
eine ausreichende Grundlage, um eine befriedigende Sicht der Ereignisse zu liefern. Zu diesen hat er zwar einen direkten Zugang, aber zugleich ist sein persönliches Interesse an der positiven Darstellung der eigenen Rolle in der Vergangenheit zu groß, als dass sich ein umsichtiger Leser ihm würde anvertrauen können. Der Leser könnte sich nur durch außertextuelle Daten Sicherheit über die tatsächliche Rolle des Protagonisten verschaffen, um nicht partiellen oder kompletten Täuschungen (man denke an den Fall Wilkomirski, an Albert Speer etc.) zu erliegen. Die Komposition der Grass'schen Novelle schließt das dadurch aus, dass der Erzähler als kritische und relativ neutrale Instanz von vornherein die Zeugenaussagen mit den Fakten konfrontiert und beurteilt, auch wenn er sie im Wortlaut an den Leser weitergibt.

Dass es aber nach wie vor äußerst problematisch bleibt, Mitglieder der Tätergemeinschaft als Opfer darzustellen, haben die heftigen Reaktionen auf den Vorleser gezeigt, die neben Kitschmotiven vor allem eine vermeintliche Verharmlosung der Schuld brandmarkten. ${ }^{40}$ Walter Kempowski ist solchen Vorwürfen von vornherein aus dem Weg gegangen, indem er in seinem Kollektiven Tagebuch sämtliche Dokumente völlig unkommentiert und in wohlausgewogener Verteilung aus allen sozialen Schichten und allen am Krieg beteiligten Parteien zusammenkomponiert hat. Angesichts der Breite des Spektrums und der Vielzahl von Stimmen wird es dem Leser unmöglich, den Autor / Redaktor mit seinen etwaigen Sympathien und Intentionen zu verorten, statt dessen scheint das Archiv selbst zu sprechen. ${ }^{41}$ Grass verfährt ähnlich, wenn er, anstatt ausschließlich die Schiffskatastrophe zu schildern, den Mord Frankfurters an Gustloff und die Biographie Marinesko einflicht

40 Siehe Adler 2002 u. Norfolk 2002. Vgl. auch den Streit um Jörg Friedrichs Buch Der Brand, zusammengefasst in: Kettenacker, Lothar (Hg.): Ein Volk von Opfern? Die neue Debatte um den Bombenkrieg 1940-45. Berlin: Rowohlt 2003.

41 Dennoch bleiben der Autor und sein Team als auswählender, ausschneidender, zusammenstellender und anordnender Wille auch hier wirksam. Schon der Impuls, über Anzeigen nach Ego-Dokumenten zu suchen und dann diese in einem eigens aufgebauten Zentrum zu katalogisieren und auszuwerten können als auktoriale Aktivitäten gewertet werden, die die definitive Form des Gesamttextes und seine Wirkung auf die Leser gestalten, auch wenn alle enthaltenen Einzelaussagen von anderen Subjekten gemacht wurden. 
und damit die Täter-Opfer-Polarität problematisiert. Tulla Pokriefke ist nach ihrer Vorgeschichte aus den Hundejahren auch alles andere als ,unschuldig'. Eberhard Lämmert hat bereits im Personal der Hundejahre die spezifisch deutschen „Phänotypen” der totalitären Epoche gesehen und hervorgehoben, dass von den hier konfigurierten Personen „fast jeder Opfer, zugleich aber auch mitverantwortlich für die Hundejahre ist, in denen sie lebten” (LÄмmERT 1996: 276). Zwar werden hier auch schon die Täter Matern und Tulla als „Opfer” gedeutet, freilich als Opfer der Machtverhältnisse, denen sie sich selbst angedient haben, als Opfer, die zugleich Gewalt ausüben und damit jener von Primo Levi so benannten „Grauzone” angehören. Grass hat in der „Danziger Trilogie” keinesfalls versucht, Gewalt und Schuld der Täter zu banalisieren, indem er sie zu Opfern umstilisierte und sein Interpret Lämmert bestätigt, dass „Täter und Opfer einander deformieren und damit, wenn auch mit sehr unterschiedlichen Spuren, gegenseitig fürs Leben zeichnen" (LÄMMERT 1996: 276).

Grass selbst hat neuerdings noch einmal betont, dass ihm Begriffe wie „Täter” und „Opfer” nicht angemessen erscheinen, ${ }^{42}$ aber auch, dass die Akzeptanz für die Innenperspektive der ,anderen Seite' eine Frage der Zeit und des Dialogs ist. ${ }^{43}$ So notwendig es sein mag, das Gedächtnis der ,eigenen' Opfer zuzulassen, kann es doch nicht ausbleiben, dass dabei in literarischen Darstellungen wie dem Der Vorleser und Im Krebsgang Interferenzen und Konkurrenzen mit den jüdischen Opfern und denen auf alliierter Seite auftreten, Opfern ,höheren Rechts', die bei auch von Grass noch nicht angemessen integriert werden konnten. Es bleibt abzuwarten, ob eine solch komplexe Aufgabe von anderen Autoren in Zukunft in einer Weise wird gelöst werden können, die Zustimmung auf ,beiden Seiten' findet.

42 „Das sind Kategorien, die ich nicht benutze.” Grass 2003.

43 „Die Zeit, die vergangen ist, der lange Zeitraum, hat die Möglichkeit, darüber nicht nur im deutschen Selbstgespräch, sondern auch mit den Polen und Russen zu reden, vergrößert." Ebd. 


\section{Literaturverzeichnis}

\section{Primärliteratur}

AMÉRY, Jean [d.i. Hans Mayer]. Jenseits von Schuld und Sühne. Bewältigungsversuche eines Überwältigten. München, dtv 1988. (1966)

Grass, Günter. Hundejahre. Werkausgabe Bd. 5. Göttingen, Steidl 1997a. (1963)

Grass, Günter. „Schreiben nach Auschwitz.” Frankfurter Poetik-Vorlesung (1990) In: G. G. Essays und Reden III. 1980-1997. Werkausgabe Bd. 16. Göttingen, Steidl 1997b. 235-256.

Grass, Günter. Mein Jahrbundert. Werkausgabe Bd. 17. Göttingen, Steidl 1999.

GrAss, Günter. „Ich erinnere mich ...” Rede im Rahmen der litauischdeutsch-polnischen Gespräche über die Zukunft der Erinnerung. Göttingen, Steidl Verlag 2001. http://www.steidl.de/grass/

Grass, Günter. Im Krebsgang. Eine Novelle. Göttingen, Steidl 2002.

Grass, Günter. „Eine Katastrophe, aber kein Verbrechen”. Interview. Der Stern 08, 2002b.

Grass, Günter. Interview. http://gabrieleweis.de/2-bldungsbits/ literaturgeschichtsbits/werk-matrialien/grass-krebsgang/grasskrebsgang-index.htm Access Juni 2002c.

KLÜGER, Ruth, weiter leben. Eine Jugend. München, dtv 1997.

Levi, Primo. Ist das ein Mensch? Die Atempause. München, Hanser 1989.

Levi, Primo. Die Untergegangenen und die Geretteten. München, Hanser 1990.

Ludwig, Emil.David und Goliath. Geschichte eines politischen Mordes. Epilog: David Frankfurter 9 Jahre später. Zürich, Posen Verlag 1945.

Semprún, Jorge. Die große Reise. Roman. Frankfurt a.M., Suhrkamp 1981.

\section{Rezensionen, Interviews, Zeitungsartikel}

Adler, Jeremy. „Die Kunst, Mitleid mit den Mördern zu erzwingen.” Süddeutsche Zeitung 20. 4. 2002. (Ursprünglich in: Times Literary Supplement) 
Assmann, Aleida. „Funke einer gesamtgesellschaftlichen Erregung. Eine Frage der Hierarchie. Leid und Schuld sind in der deutschen Erinnerung keineswegs so unvereinbar wie es scheint." FR 3. 2. 2004

FranZen, Günter. „Der alte Mann und sein Meer.” Die Zeit. Nr. 72002.

GrAss, Günter. ,,Wir waren keine Hellseher.” Interview mit Wulf Segebrecht. FAZ 22. 12. 2003.

GüntNer, Joachim. „Opfer und Tabu. Günter Grass und das Denken im Trend." NZZ 23. 2. 2002.

Hage, Volker. „Es war das eigentlich Nicht-Mögliche” In: Der Spiegel 22. 7. 2003

(kho). „Unsere Veteranen. Grass stößt in Königsberg auf Kritik.” FAZ 13. 6. 2003.

KüCHEMAnN, Fridtjof. „Aufzeichnungen eines Einsiedlerkrebses.” FAZ 5. 2. 2002.

Mangold, Ijoma. „Jedes Leid hat ein Recht auf Erlösung.” SZ 20. 2. 2002.

RiTTER, Henning. „Der Weg vom historischen Dokument zu einem literarischen Zeugnis." FAZ 20. 2. 2002.

SEIDL, Claudius. „Die Härte der späten Geburt.” FAZ 20. 2. 2002.

SpIEGEL, Hubert. „Das mußte aufschraiben!” FAZ 9. 2. 2002

\section{Sekundärliteratur}

Adorno, Theodor W. Gesammelte Schriften. Frankfurt a. M., Suhrkamp 1986. [Digitale Bibliothek Bd. 97, Berlin: Directmedia 2003]

Agamben, Giorgio. Remnants of Auschwit: The Witness and the Archive. New York: Zone Books 1999.

Assmann, Jan. „Gedächtnis”. In: Stefan Jordan (Hg.): Lexikon Gescbichtswrissenschaft. 100 Grundbegriffe. Stuttgart, Reclam 2002, 97-101.

Assmann, Jan. Das kulturelle Gedächtnis. Schrift, Erinnerung und politische Identität in frühen Hochkulturen. München, Beck 1997. 
BEYER, Marcel. „Das wilde Tier im Kopf des Historikers.” In: St. Deines u.a. (Hg.): Historisierte Subjekte - Subjektivierte Historie. Zur Verfügbarkeit und Unverfügbarkeit von Geschichte. Berlin, de Gruyter 2003, 295-301.

BOHLEBER, Werner. „Transgenerationelles Trauma, Identifizierung und Geschichtsbewusstsein”. In: Jörn Rüsen / Jürgen Straub (Hg.). Die dunkle Spur der Vergangenheit. Psychoanalytische Zugänge zum Geschichtsbewnsstsein, Erinnerung, Geschichte, Identität 2. Frankfurt a.M., Suhrkamp 1998, 256-274.

DiNER, Dan. Kreisläufe. Nationalsozialismus und Gedächtnis. Berlin, Berlin Verlag 1995.

DöBLIN, Alfred. „Der historische Roman und wir”. In: A.D.: Aufsätz̨e zur Literatur. Olten, Freiburg, Walter 1963.

Friedlander, Saul (Hg.). Probing the Limits of Representation. Nazism and the "Final Solution”. Cambridge / Mass., London, Harvard University Press 1992.

Galde, Helmut. „’ ... Verdadero como solo puede serlo la ficción.' Un ficticio texto testimonial en yiddish y sus implicaciones para la referencialidad de la literatura." Inter Litteras. no. 9 2001a, 51-73.

Galde, Helmut. „Zeugnis oder Konstruktion? ,Referentielle Rhetorik“ und Rezeption von ,Authentizität ${ }^{6}$ anhand von Jakob Littners und Wolfgang Koeppens Ghettobericht.” In: Brückenschlag. Caracas 2002a, 184-195.

GALLE, Helmut. „Das Bild des Nazitäters in der deutschen Nachkriegsliteratur.” Vortrag. Hannover 2002b.

Gadie, Helmut. „Das Subjekt angesichts des Äußersten. Zeugnisse von Holocaust-Opfern als Dokumente für die Widerständigkeit von Subjektivität. In: Stefan Deines, Stephan Jaeger \& Ansgar Nünning (Hg.): Historisierte Subjekte - Subjektivierte Historie. Zur Verfügbarkeit und Unverfügbarkeit von Geschichte. Berlin / New York, de Gruyter 2003, 161-183.

GenETTE, Gérard. Die Erzählung. Stuttgart, UTB 1998.

GeNeTte, Gérard. Fiktion und Diktion. München, Fink 1992.

Hamburger, Käte. Die Logik der Dichtung. Stuttgart, Klett 1957.

Huyssen, Andreas. „Transnationaler Holocaustdiskurs und lateinamerikanische Diktaturerfahrung." In: Bolle, W., Galle, H. (Hg.): Blickwechsel. Band 1. São Paulo: Edusp / Monferrer Prod. 2005. 258-278. 
LÄMmERT, Eberhard. „Geschichten von der Geschichte. Geschichtsschreibung und Geschichtsdarstellung im Roman.” In: Poetica 17 (1985), 228254.

LÄMmERT, Eberhard. „GULAG Europa. Aleksandr Solschenizyn, Der Archipel GULAG, Günter Grass, Hundejahre, Andrzej Szczypiorski, Die schöne Frau Seidenman.” In: E.L. u. B. Naumann (Hg.): Wer sind wir? Europäische Phänotypen im Roman des 20. Jabrbunderts. München, W. Fink 1996, 265284.

MAZZARI, Marcus Vinicius. "Formas de Integração entre Ficção e História." In: M.M.: Romance de Formação em Perspectiva Histórica. O Tambor de Lata de G. Grass. São Paulo, Ateliê 1999.

MüLLER, Harro. „Stalingrad und kein Ende. Zur Präsentation des Zweiten Weltkriegs in drei historischen Romanen.” In: Wolfgang Küttler / Jörn Rüsen / Ernst Schulin (Hg.): Geschichtsdiskurs Bd. 5: Globale Konflikte, Erinnerungsarbeit und Neurorientierung seit 1945. Frankfurt a.M., Fischer 1999, 297-313.

NeuHaus, Volker. Schreiben gegen die verstreichende Zeit. Zu Leben und Werk von Günter Grass. München: dtv 1997.

Nietzsche, Friedrich. „Vom Nutzen und Nachteil der Historie für das Leben". Werke in drei Bänden. Hg. v. Karl Schlechta. München, Hanser 1954, Bd. 1, 209-286.

RüsEN, Jörn. Zerbrechende Zeit. Über den Sinn der Geschichte. Köln, Weimar, Wien, Böhlau 2001.

SCHIEDER, Theodor (Hg.). Dokumentation der Vertreibung der Deutschen aus OstMitteleuropa. Bd. 1 Die Vertreibung der deutschen Bevölkerung aus den Gebieten östlich der Oder-Neiße. München 1984.

SCHLINK, Bernhard. Vergangenheitsschuld und gegenwärtiges Recht. Frankfurt a.M., Suhrkamp 2002. 